GTME7 v2

\title{
The Geometrization of Maxwell's Equations and the Emergence of Gravity
}

\section{Raymond J. Beach}

Lawrence Livermore National Laboratory, L-465, 7000 East Avenue, Livermore, CA 94551

E-mail: beach2@IInl.gov

ORCID (iD) 0000-0002-4099-130X

\section{ABSTRACT}

A recently proposed classical field theory in which Maxwell's equations are replaced by an equation that couples the Maxwell tensor to the Riemann-Christoffel curvature tensor in a fundamentally new way is reviewed and extended. This proposed geometrization of the Maxwell tensor provides a succinct framework for the classical Maxwell equations which are left intact as a derivable consequence. Beyond providing a basis for the classical Maxwell equations, the coupling of the Riemann-Christoffel curvature tensor to the Maxwell tensor leads to the emergence of gravity, with all solutions of the proposed theory also being solutions of Einstein's equation of General Relativity augmented by a term that can mimic the properties of dark matter and/or dark energy. Both electromagnetic and gravitational phenomena are put an equal footing with both being tied to the curvature of space-time. Using specific solutions to the proposed theory, the unification brought to electromagnetic and gravitational phenomena as well as the consistency of solutions with those of the classical Maxwell and Einstein field equations is emphasized throughout. Unique to the proposed theory and based on specific solutions are the emergence of antimatter and its behavior in gravitational fields, the emergence of dark matter and dark energy mimicking terms in the context of General Relativity, an underlying relationship between electromagnetic and gravitational radiation, and the impossibility of negative mass solutions that would generate repulsive gravitational fields or antigravity. Finally, a method for quantizing the charge, mass, and angular momentum of particle-like solutions as well as the possibility of superluminal transport of specific curvature related quantities is conjectured.

Keywords: Maxwell's equations, General Relativity, unification of electromagnetism and gravity, dark matter and dark energy, electromagnetic and gravitational radiation, antimatter, antigravity, quantization, superluminal transport 
LLNL-JRNL-726138

\section{INTRODUCTION}

Electromagnetic and gravitational fields have long range interactions characterized by speed of light propagation; similarities that suggest these fields should be coupled together at the classical physics level. Although this coupling or unification is a well-worn problem with many potential solutions having been proposed, it is fair to say that there is still no generally accepted classical field theory that can explain both electromagnetism and gravitation in a coupled or unified framework. ${ }^{[1]}$ Today, the existence of electromagnetic and gravitational fields are generally understood to be distinct and independent, with electromagnetic fields described by Maxwell's equations, and gravitational fields described by Einstein's equation of General Relativity. The purpose of this manuscript is to assess a recently proposed set of field equations that introduce a geometric description of electromagnetism and from which gravitation then emerges.

Assuming the geometry of nature is Riemannian with four dimensions, the three field equations in Table I are proposed to provide a description of classical physics at the level of the Maxwell and Einstein Field Equations (M\&EFEs) ${ }^{[2]}$ but to then to go further by geometrically unifying electromagnetism and gravitation.

Table I. Fundamental field equations of proposed theory

\begin{tabular}{|c|c|}
\hline Equations & $\begin{array}{c}\text { Equation } \\
\text { number }\end{array}$ \\
\hline$F_{\mu \nu ; \kappa}=a^{\lambda} R_{\lambda \kappa \mu v}$ & (1) \\
\hline$u^{\lambda} u_{\lambda}=-1$ & (2) \\
\hline$\left(\rho_{m} u^{\mu} u^{v}+F_{\lambda}^{\mu} F^{v \lambda}-\frac{1}{4} g^{\mu v} F^{\rho \sigma} F_{\rho \sigma}\right)_{; v}=0$ & (3) \\
\hline
\end{tabular}

In Table I, $F_{\mu \nu}$ is the Maxwell tensor, $g_{\mu v}$ is the metric tensor, $R_{\lambda \mu v \kappa}$ is the Riemann-Christoffel (R-C) curvature tensor, $u^{\lambda}$ is the four-velocity, and $\rho_{m}$ is the mass density. Importantly, equations (2) and (3) are not new, both being well accepted foundational equations of classical physics with (2) the normalization of the four-velocity $u^{\lambda}$, and (3) the conservation of energy and momentum for matter and 
electromagnetic fields. Only equation (1), which couples the derivatives of the Maxwell tensor to the RC tensor through a vector field $a^{\lambda}$ is new.

Collectively, the equations of Table I are very close to the foundational equations of classical electromagnetism. In fact, if equation (1) were to be replaced by Maxwell's equations, then the equations of Table I would correspond exactly to the foundational equations of classical electromagnetism. In section 2.1 it will be shown that the classical Maxwell equations are a derivable consequence of equation (1) and the properties of the R-C tensor, a result that ensures the field theory based on equations (1) through (3) encompass all the equations of classical electromagnetism. It is in this sense that equation (1) can be thought of as a geometricized version of Maxwell's equations.

The goal of this manuscript is to show through an axiomatic development that the continuous field theory based on fundamental field equations (1) through (3) covers classical electromagnetic and gravitational phenomena in a unified manner. In the view of the proposed theory, both electromagnetic and gravitational phenomena are put on an equal footing with both being intimately tied to the curvature of space-time. Additionally, it will be shown that the description of gravity that emerges is consistent with Einstein's equation of General Relativity augmented by a term that can mimic the properties of dark matter and/or dark energy.

Listed in Table II are the continuous field variables that the proposed field theory's fundamental equations solve for. Except for $a^{\lambda}$, all the field variables listed in Table II are familiar to classical physics.

Table II. Dynamic fields

\begin{tabular}{|c|l|}
\hline Field & \multicolumn{1}{c|}{ Description } \\
\hline$g_{\mu v}$ & Metric tensor \\
\hline$F_{\mu v}$ & Maxwell tensor \\
\hline$u^{\lambda}$ & Four-velocity vector field \\
\hline$\rho_{m}$ & $\begin{array}{l}\text { Mass density scalar field } \\
\text { (Rest value in a locally inertial frame) }\end{array}$ \\
\hline$a^{\lambda}$ & Four-vector coupling electromagnetism to gravitation \\
\hline
\end{tabular}

Just as it does in classical continuum physics, the four-velocity vector field $u^{\lambda}$ describes the motion of the mass density field $\rho_{m}$. However, unlike the situation in classical physics where $\rho_{m}$ is treated as an external 
GTME7 v2

4

LLNL-JRNL-726138

field, here it is treated as a dynamic field that is solved for as part of any solution to fundamental equations (1) through (3). For a classical field theory, this treatment of $\rho_{m}$ as a dynamic field results in several interesting predictions: the emergence of antimatter and its behavior in gravitational fields, the emergence of dark matter and dark energy mimicking terms in the context of General Relativity, an underlying relationship between electromagnetic and gravitational radiation, and the impossibility of negative mass solutions that would generate repulsive gravitational fields or antigravity. All these points are brought out through specific solutions investigated in section 4 of this manuscript.

Throughout the manuscript geometric units are used with a metric tensor having signature $[+,+,+,-]$. Spatial indices run from 1 to 3 with 4 the time index. The notation within uses commas before tensor indices to indicate ordinary derivatives and semicolons before tensor indices to indicate covariant derivatives. For the definitions of the R-C curvature tensor and the Ricci tensor, the conventions used by Weinberg are followed. ${ }^{[3]}$

\section{CONSEQUENCES OF THE FUNDAMENTAL FIELD EQUATIONS}

\subsection{The equations of electromagnetism}

In the conventional theoretical derivations of the classical equations of electromagnetism, Maxwell's homogeneous equation must be taken as an axiom so that the vector potential $A_{\mu}$ can be introduced. The introduction of $A_{\mu}$ is necessary so that a Lagrangian consisting of a matter term, a free electromagnetic field term and a matter-field interaction term can be defined and written in terms of $A_{\mu}$ . Maxwell's inhomogeneous equation and the Lorentz force law are then derived using a stationary-action calculation in which $A_{\mu}$ and the spatial positions of masses and charges are treated as the dynamic variables. ${ }^{4}$ Finally, the vanishing of the variation of the scalar action with respect to $g_{\mu v}$ is used to define the conserved energy-momentum tensor given in equation (3). All of these equations of classical electromagnetism are also a direct result of fundamental field equations (1) through (3). The only difference between the set of equations derived using the classical arguments just outlined here and their derivation to be given in what follows is that Maxwell's homogeneous equation is no longer taken as an axiom in the proposed theory but rather is replaced by fundamental equation (1) as an axiom, with all of Maxwell's equations then becoming a derived consequence of (1) and the properties of the R-C tensor. 
Here I give a short description of the derivation of the classical equations of electromagnetism in the framework of the proposed theory. The point in going through this purely formal development is to show that the classical equations of electromagnetism are derivative only to the fundamental field equations (1) through (3) and the algebraic properties of the R-C tensor.

To begin, the antisymmetry of $F_{\mu \nu}$ follows directly from equation (1) and the algebraic property of the RC tensor,

$$
R_{\lambda \kappa \mu \nu}=-R_{\lambda \kappa \nu \mu}
$$

Next, I derive Maxwell's homogeneous equation using the algebraic property of the R-C tensor,

$$
R_{\lambda \kappa \mu v}+R_{\lambda \mu v \kappa}+R_{\lambda v \kappa \mu}=0
$$

which on contracting with $a^{\lambda}$ gives,

$$
a^{\lambda} R_{\lambda \kappa \mu v}+a^{\lambda} R_{\lambda \mu v \kappa}+a^{\lambda} R_{\lambda v \kappa \mu}=0
$$

Comparing (6) and (1) then gives,

$$
\begin{gathered}
F_{\mu \nu ; \kappa}+F_{v \kappa ; \mu}+F_{\kappa \mu ; \nu}=0 \\
o r \\
F_{\mu v, \kappa}+F_{v \kappa, \mu}+F_{\kappa \mu, \nu}=0
\end{gathered}
$$

thus, establishing Maxwell's homogeneous equation, and where the switch from the covariant to ordinary derivatives in (7) is justified by the antisymmetry of $F_{\mu v}$. Having established the antisymmetry of $F_{\mu \nu}$ and the vanishing of its anti-symmetrized derivative, which is just a statement of Maxwell's homogeneous equation (7), the converse of Poincaré's lemma establishes that $F_{\mu \nu}$ can itself be expressed as the antisymmetrized derivative of a vector function,

$$
F_{\mu v}=A_{v, \mu}-A_{\mu, v}
$$

where $A_{\mu}$ is the classical electromagnetic vector potential. Because $F_{\mu \nu}$ can be expressed as the antisymmetrized derivative of the vector potential $A_{\mu}$, its value will be unaffected by a gauge transformation in which a gradient field is added to $A_{\mu}$, 
GTME7 v2

$$
A_{\mu} \rightarrow A_{\mu}+\partial_{\mu} \varphi
$$

Next, Maxwell's inhomogeneous equation and the definition of charge density in the proposed theory are derived. Starting with equation (1) and contracting the $\mu$ and $\kappa$ indices gives,

$$
F_{; \mu}^{\mu v}=a^{\lambda} R_{\lambda \mu}^{\mu v}=-a^{\lambda} R_{\lambda}^{v}
$$

If the vector field $a^{\lambda} R_{\lambda}{ }^{v}$ which appears on the RHS of $(10)$ is time-like,

$$
\left(a^{\lambda} R_{\lambda}^{v}\right)\left(a^{\sigma} R_{\sigma v}\right)<0
$$

then it can be equated to the product of a scalar quantity and its associated four-velocity. For example, the following identification could be made,

$$
\rho_{c} u^{v}=a^{\lambda} R_{\lambda}^{v}
$$

To see that this defining procedure is always legitimate when (11) is satisfied, one can solve (12) for $\rho_{c}$ by taking the magnitude of each side,

$$
\rho_{c}^{2}=-a^{\lambda} R_{\lambda}^{v} a^{\sigma} R_{\sigma v}
$$

As long as (11) is satisfied, the RHS of (13) is guaranteed to be nonnegative always with $\rho_{c}$ given by,

$$
\rho_{c}= \pm \sqrt{-a^{\lambda} R_{\lambda}^{v} a^{\sigma} R_{\sigma V}}
$$

With the exception of section 5.7, equation (11) constraining $a^{\lambda} R_{\lambda}{ }^{v}$ to be time-like will be assumed true in this manuscript. Under this constraint, equation (12) then serves as the definition of charge density $\rho_{c}$ in terms of the fundamental fields listed in Table II, with its value given by (14) in terms of $a^{\lambda}$ and the Ricci tensor. An alternative expression for $\rho_{c}$ which also follows from (12), and which will be useful when finding specific solutions to the proposed theory is,

$$
\rho_{c}=-u^{v} a^{\lambda} R_{\lambda v}
$$

With the definition of $\rho_{c}$ given in (12), Maxwell's inhomogeneous equation,

$$
F_{; \mu}^{\mu v}=-\rho_{c} u^{v}
$$


follows from (10). The preceding establishes that Maxwell's classical field equations and the concept and definition of charge density are all derivable consequences of equations (1) and (2) under the assumption that $a^{\lambda} R_{\lambda}{ }^{v}$ is time-like. Next, I derive the conservation of charge. Returning to the antisymmetry of $F_{\mu \nu}$, it follows that,

$$
F_{; \mu ; \nu}^{\mu \nu} \equiv 0
$$

which is an identity for all antisymmetric tensors. Comparing (17) to the contracted derivative of Maxwell's inhomogeneous equation (16) then gives,

$$
F_{; \mu ; v}^{\mu v}=0=-\left(\rho_{c} u^{v}\right)_{; \nu},
$$

thus, establishing the conservation of charge,

$$
\left(\rho_{c} u^{v}\right)_{; v}=0
$$

Consider next the Lorentz force law and the conservation of mass, both of which are derivative to the conserved energy-momentum tensor given in equation (3). To see this, distribute the covariant derivative in (3),

$$
\left(\rho_{m} u^{v}\right)_{; \nu} u^{\mu}+\rho_{m} u_{; \nu}^{\mu} u^{v}+F^{\mu \lambda} F_{\lambda ; \nu}^{v}+F_{; \nu}^{\mu \lambda} F_{\lambda}^{v}-\frac{1}{2} g^{\mu v} F^{\rho \sigma} F_{\rho \sigma ; v}=0
$$

With some substitutions and rearrangements using Maxwell's homogeneous equation (7) and inhomogeneous equation (16), (20) can be re written as,

$$
\left(\rho_{m} u^{v}\right)_{; \nu} u^{\mu}+\rho_{m} u_{; \nu}^{\mu} u^{v}-\rho_{c} F_{\lambda}^{\mu} u^{\lambda}=0
$$

Contracting (21) with $u_{\mu}$, the $2^{\text {nd }}$ and $3^{\text {rd }}$ terms on the LHS of (21) are zeroed due to (2) and the antisymmetry of $F_{\mu \nu}$, respectively, leaving,

$$
\left(\rho_{m} u^{v}\right)_{; v}=0
$$

thus, establishing the conservation of mass equation. Using (22) to zero out the conservation of mass term in (21) then leaves the Lorentz force law, 


$$
\rho_{m} \frac{D u^{\mu}}{D \tau}=\rho_{c} F_{\lambda}^{\mu} u^{\lambda}
$$

where $\frac{D u^{\mu}}{D \tau} \equiv u_{; \lambda}^{\mu} u^{\lambda}$

In summary, the theory of electromagnetism being proposed here in no way changes the traditional equations of classical electromagnetism except that Maxwell's homogeneous equation is no longer taken as an axiom but is rather a derived consequence of fundamental field equation (1) and the properties of the R-C tensor. However, adopting equation (1) as a starting point does introduce conceptual changes in electromagnetic phenomenology that go beyond the predictions of classical electromagnetism. For example, the charge density $\rho_{c}$ is no longer an externally inserted field as it is in the classical physics picture. This can be seen in the identification given in (14) where the scalar charge density is shown to be dependent on the vector field $a^{\lambda}$ and the Ricci tensor. In subsequent sections, the proposed theory based on fundamental equations (1) through (3) will be developed further using specific solutions to show that electromagnetic and gravitational phenomena are put on an equal footing, with both being intimately tied to nonzero space-time curvatures. The cost of this unification is the introduction of the vector field $a^{\lambda}$, a field not known to traditional classical physics but as will be shown in section 3.3 is not completely new either, being directly relatable to the familiar vector potential $A_{v}$ of classical electromagnetism.

\subsection{A theory of gravitation}

While evident from the preceding discussion where the equations of classical electromagnetism were shown to follow from fundamental field equations (1) through (3) and the assumption that $a^{\lambda} R_{\lambda}{ }^{v}$ is timelike, at this point it is not obvious that the same can be said of Einstein's equation of General Relativity,

$$
G^{\mu v}=-8 \pi T^{\mu v}
$$

where $G^{\mu v} \equiv R^{\mu v}-\frac{1}{2} g^{\mu v} R$ is the Einstein tensor. As will be shown, the particle-like solution to be analyzed in section 4.1 demonstrates that the Reissner-Nordström metric is an exact solution of the fundamental field equations (1) through (3), thus establishing that the proposed theory and classical General Relativity (24) support the same metric field solutions, at least in the case of spherical symmetry. However, one must go further to determine if Einstein's field equation is a derivable consequence of the fundamental equations (1) through (3). 
To investigate this issue, I start by considering the conserved energy-momentum tensor (3). An immediate consequence of $G^{\mu v}$ and $T^{\mu v}$ being both symmetric and independently conserved, independently conserved because $G_{; v}^{\mu v}=0$ by the Bianchi identity and $T_{; v}^{\mu v}=0$ by equation (3), is that for any constant $\alpha$, one can define a tensor field $\Lambda^{\mu v}$ by,

$$
\Lambda^{\mu v} \equiv G^{\mu v}-\alpha T^{\mu v}
$$

With this definition for $\Lambda^{\mu v}$, it is constrained to be both symmetric,

$$
\Lambda^{\mu v}=\Lambda^{v \mu}
$$

and conserved,

$$
\Lambda_{; v}^{\mu v}=0
$$

The value of the constant $\alpha$ in (25) is completely arbitrary and without physical significance because $\Lambda^{\mu v}$ as defined can absorb any change in $\alpha$ such that (25) remains satisfied. Taking advantage of this arbitrariness and setting the value of the constant $\alpha=-8 \pi$ then gives with a slight rearrangement of (25),

$$
G^{\mu v}=-8 \pi T^{\mu v}+\Lambda^{\mu v}
$$

which is recognized as Einstein's equation of General Relativity (24) augmented on its RHS by the term $\Lambda^{\mu v}$. From the perspective of classical General Relativity, $\Lambda^{\mu v}$ mimics the properties of dark matter and/or dark energy, viz., it is a conserved and symmetric tensor field, it is a source of gravitational fields in addition to $T^{\mu v}$, and it has no interaction signature beyond the gravitational fields it sources.

It is important to recognize at this point in the development of the proposed theory that (28) is a trivial result with no physical significance. Any solution of the fundamental field equations (1) through (3) must necessarily be a solution of (28) for some choice $\Lambda^{\mu v}$. In fact, the validity of (28) rests only on the existence of a conserved energy-momentum tensor and the properties of the R-C tensor, and so will be true in any physical theory that has a conserved energy-momentum tensor. However, the interesting point in the context of the proposed theory is that the value of $\Lambda^{\mu v}$ can be calculated in terms of the dynamic field variables given in Table II, and without recourse to postulating the existence of other exotic 
forms of matter or energy. This feature will be investigated further in subsequent sections in which specific solutions to the fundamental field equations are developed.

In the view of the proposed theory, gravitation emerges as a manifestation of the geometricized theory of electromagnetism based on field equations (1) through (3). While this geometricized theory of electromagnetism encompass all of the classical equations of electromagnetism, it does start with a different set of foundational equations, specifically with Maxwell's equations being replaced by equation (1). It is the coupling of the derivatives of the Maxwell tensor to the R-C tensor in (1) that brings gravitation into the picture. While viewing gravitation as a manifestation of electromagnetism and vice versa is not new, ${ }^{[5],[6],[7],[8],[9]}$ the specific approach being followed here with equation (1) is fundamentally new.

\section{MATHEMATICAL STRUCTURE OF FUNDAMENTAL FIELD EQUATIONS}

\subsection{Symmetries of fundamental field equations}

Three important global symmetries of the fundamental field equations (1) through (3) that are shared by all their solutions are reviewed here. The first of these global symmetries corresponds to chargeconjugation,

$$
\left(\begin{array}{c}
u^{\lambda} \\
a^{\lambda} \\
F^{\mu v} \\
g_{\mu v} \\
\rho_{c} \\
\rho_{m}
\end{array}\right) \rightarrow\left(\begin{array}{c}
u^{\lambda} \\
-a^{\lambda} \\
-F^{\mu v} \\
g_{\mu v} \\
-\rho_{c} \\
\rho_{m}
\end{array}\right)
$$

the second corresponds to a matter-antimatter transformation as will be discussed in section 5.4,

$$
\left(\begin{array}{c}
u^{\lambda} \\
a^{\lambda} \\
F^{\mu v} \\
g_{\mu v} \\
\rho_{c} \\
\rho_{m}
\end{array}\right) \rightarrow\left(\begin{array}{c}
-u^{\lambda} \\
-a^{\lambda} \\
-F^{\mu v} \\
g_{\mu v} \\
\rho_{c} \\
\rho_{m}
\end{array}\right)
$$


LLNL-JRNL-726138

and the third to the product of the first two,

$$
\left(\begin{array}{c}
u^{\lambda} \\
a^{\lambda} \\
F^{\mu v} \\
g_{\mu v} \\
\rho_{c} \\
\rho_{m}
\end{array}\right) \rightarrow\left(\begin{array}{c}
-u^{\lambda} \\
a^{\lambda} \\
F^{\mu v} \\
g_{\mu v} \\
-\rho_{c} \\
\rho_{m}
\end{array}\right) .
$$

All three transformations leave the fundamental equations (1) through (3) unchanged. Adding the identity transformation to these symmetries forms the Klein-4 group, with the product of any two of the symmetries (29) through (31) giving the remaining symmetry.

Note that among the fundamental fields of the theory, only $g_{\mu v}$ and $\rho_{m}$ are unchanged by all the symmetry transformations, a fact that will be useful in section 5.6 for defining boundary conditions that lead to quantized mass, charge, and angular momentum of particle-like solutions as well as for the treatment of antimatter. Finally, in addition to the proposed theory's general covariance and global symmetries (29) through (31), it also exhibits the electromagnetic gauge covariance of classical electromagnetism as detailed in equations (8) and (9).

\subsection{Relationship of $a^{\lambda}$ to the classical electromagnetic vector potential $A^{\lambda}$}

The only new piece of physics in the foregoing development has been the introduction of equation (1) and the vector field $a^{\lambda}$, a field that has no counterpart in the conventionally accepted development of classical physics but here serves to couple through (1), the derivatives of the Maxwell tensor to the R-C tensor. In some respects, $a^{\lambda}$ plays a role similar the conventional vector potential $A^{\lambda}$ of classical electromagnetism, and in fact can be directly related to it. To see this, take the covariant derivative of both sides of (8) giving,

$$
F_{\mu v ; \kappa}=A_{\nu ; \mu ; \kappa}-A_{\mu ; ; ; \kappa}
$$

Now compare (32) to equation (1) rewritten as,

$$
F_{\mu \nu ; \kappa}=a^{\lambda} R_{\lambda \kappa \mu v}=-a_{\kappa ; \mu ; v}+a_{\kappa ; ; ; \mu}
$$


where the RHS of (33) follows from the commutation property of covariant derivatives. Equating the RHS'S of equations (32) and (33) gives,

$$
a_{\kappa ; v ; \mu}-a_{\kappa ; \mu ; \nu}=A_{v ; \mu ; \kappa}-A_{\mu ; \nu ; \kappa}
$$

establishing a connection between $a^{\lambda}$ and $A^{\lambda}$.

\subsection{A system of first order partial differential equations - Do solutions exist?}

Equation (1) represents a mixed system of first order partial differential equations for $F_{\mu \nu}$ and illustrates one of the mathematical complexities of the fundamental field equations (1) through (3) that must be dealt with when attempting to find solutions. ${ }^{[10]}$ Specifically, mixed systems of first order partial differential equations must satisfy integrability conditions if solutions are to exist. ${ }^{[11]}$ Although there are several ways of stating what these integrability conditions are, perhaps the simplest is given by,

$$
F_{\mu v ; \kappa ; \lambda}-F_{\mu v ; \lambda ; \kappa}=-F_{\mu \sigma} R_{\nu k \lambda}^{\sigma}-F_{\sigma v} R_{\mu \kappa \lambda}^{\sigma}
$$

which can be derived using the commutation relations for covariant derivatives. Using (1) to substitute for $F_{\mu v ; \kappa}$ in (35) gives,

$$
\left(a^{\rho} R_{\rho \kappa \mu \nu}\right)_{; \lambda}-\left(a^{\rho} R_{\rho \lambda \mu \nu}\right)_{; k}=-F_{\mu \sigma} R_{\nu k \lambda}^{\sigma}-F_{\sigma \nu} R_{\mu \kappa \lambda}^{\sigma}
$$

which can be interpreted as conditions that are automatically satisfied by any solution consisting of expressions for $g_{\mu \nu}, a^{\lambda}$ and $F_{\mu \nu}$ that satisfy (1). With (36) as integrability conditions that must be satisfied by any solution of (1), the question that naturally arises is this: Are these integrability conditions so restrictive that perhaps no solution to the proposed theory exists? Although this view could be construed as making the proposed field theory uninteresting because perhaps no solutions exist, it will be shown that solutions that are consistent with known solutions of the classical M\&EFEs can be found. Additionally, equation (36) which is linear in $F_{\mu \nu}$ is often useful in developing solutions to the full set of field equations (1) through (3), an approach that will be used in the solution to be found in section 4.1. Finally, to further elucidate questions regarding solutions of the proposed theory, an outline showing how the field equations can be solved numerically is given in the appendix where an analysis is presented of fundamental equations (1) through (3) in terms of a Cauchy initial value problem. 
LLNL-JRNL-726138

\section{SOLUTIONS TO FUNDAMENTAL FIELD EQUATIONS}

\subsection{Spherically symmetric charged particle}

In this section a solution representing a non-rotating, spherically symmetric charged mass is investigated. It is demonstrated that the Reissner-Nordström metric with an appropriate choice for the fields $F_{\mu \nu}, a^{\lambda}, u^{\lambda}, \rho_{c}$ and $\rho_{m}$ satisfies the fundamental field equations (1) through (3). Although the presentation in this section is purely formal, it is included here for several reasons. First, if the theory could not describe the asymptotic electric and gravitational fields of a charged particle it would be of no interest on physical grounds. Second and as already discussed, the presented theory requires the solution of a mixed system of first order partial differential equations, a system that may be so restrictive that no solutions exist and so an outline of at least one methodology to a solution is warranted. Finally, having an exact solution to field equations (1) through (3) that corresponds to a known solution of the M\&EFEs enables a direct comparison and contrast of the two solutions to be made.

To proceed, I draw on a solution for a spherically symmetric charged particle that was previously derived. ${ }^{[12]}$ Starting with the Reissner-Nordström metric ${ }^{[13]}$,

$$
g_{\mu \nu}=\left(\begin{array}{cccc}
\frac{1}{1+\frac{q^{2}}{r^{2}}-\frac{2 m}{r}} & 0 & 0 & 0 \\
0 & r^{2} & 0 & 0 \\
0 & 0 & r^{2} \operatorname{Sin}^{2}(\theta) & 0 \\
0 & 0 & 0 & -1-\frac{q^{2}}{r^{2}}+\frac{2 m}{r}
\end{array}\right)
$$

the Ricci tensor that follows from (37),

$$
R_{\lambda}{ }^{v}=\frac{q^{2}}{r^{4}}\left(\begin{array}{cccc}
1 & 0 & 0 & 0 \\
0 & -1 & 0 & 0 \\
0 & 0 & -1 & 0 \\
0 & 0 & 0 & 1
\end{array}\right)
$$

and a guess for $a^{\lambda}$,

$$
a^{\lambda}=\left(0,0,0, c_{1}\right)
$$

where $c_{1}$ is a yet to be determined constant, $\rho_{c}$ can be determined from (14) as, 


$$
\rho_{c}= \pm \frac{q^{2} \sqrt{q^{2}+r(r-2 m)}}{r^{5}}\left|c_{1}\right|
$$

Using equation (12), $u^{\lambda}$ is then found to be,

$$
u^{\lambda}=\left(0,0,0, \pm \frac{r}{\sqrt{q^{2}+r(r-2 m)}} \frac{c_{1}}{\left|c_{1}\right|}\right)
$$

The next step is to satisfy (1) by solving for $F_{\mu \nu}$. Rather than tackle this head on by directly trying to find a solution to the mixed system of first order partial differential equations (1), I instead solve the integrability equations (36) which are linear in $F_{\mu v}$ for $F_{\mu v}$. Proceeding in this manner I find that all the integrability equations are satisfied for $F_{\mu \nu}$ given by,

$$
F_{\mu \nu}=\left(\begin{array}{cccc}
0 & B_{\phi} & -B_{\theta} & E_{r} \\
-B_{\phi} & 0 & B_{r} & E_{\theta} \\
B_{\theta} & -B_{r} & 0 & E_{\phi} \\
-E_{r} & -E_{\theta} & -E_{\phi} & 0
\end{array}\right)=\left(\begin{array}{cccc}
0 & 0 & 0 & \frac{\left(m r-q^{2}\right)}{r^{3}} c_{1} \\
0 & 0 & 0 & 0 \\
0 & 0 & 0 & 0 \\
-\frac{\left(m r-q^{2}\right)}{r^{3}} c_{1} & 0 & 0 & 0
\end{array}\right)
$$

By direct substitution it is easily verified that $F_{\mu \nu}$ as given in (42) is a solution of (1). ${ }^{[14]}$ Choosing the value of the undetermined constant $c_{1}= \pm q / \mathrm{m}$ then gives an electric field which agrees with the Coulomb field of a point charge to leading order in $1 / r$. Finally, the remaining unknown field, the scalar mass density field $\rho_{m}$ is found using the conserved energy-momentum tensor (3). Substituting the known fields into (3) and then solving for $\rho_{m}$ gives,

$$
\rho_{m}=\frac{q^{4}\left(q^{2}-2 m r+r^{2}\right)}{m^{2} r^{6}}
$$

To summarize, the following expressions for $g_{\mu \nu}, F_{\mu \nu}, a^{\lambda}, u^{\lambda}, \rho_{c}$ and $\rho_{m}$ are an exact solution to the fundamental field equations (1) through (3): 


$$
\begin{aligned}
& g_{\mu \nu}=\left(\begin{array}{cccc}
\frac{1}{1-\frac{2 m}{r}+\frac{q^{2}}{r^{2}}} & 0 & 0 & 0 \\
0 & r^{2} & 0 & 0 \\
0 & 0 & r^{2} \operatorname{Sin}^{2}(\theta) & 0 \\
0 & 0 & 0 & -\left(1-\frac{2 m}{r}+\frac{q^{2}}{r^{2}}\right)
\end{array}\right) \\
& F_{\mu \nu}=\left(\begin{array}{cccc}
0 & B_{\phi} & -B_{\theta} & E_{r} \\
-B_{\phi} & 0 & B_{r} & E_{\theta} \\
B_{\theta} & -B_{r} & 0 & E_{\phi} \\
-E_{r} & -E_{\theta} & -E_{\phi} & 0
\end{array}\right)=s\left(\begin{array}{cccc}
0 & 0 & 0 & \frac{q}{r^{2}}-\frac{q^{3} / m}{r^{3}} \\
0 & 0 & 0 & 0 \\
0 & 0 & 0 & 0 \\
-\frac{q}{r^{2}}+\frac{q^{3} / m}{r^{3}} & 0 & 0 & 0
\end{array}\right) \\
& u^{\lambda}=s\left(0,0,0, \frac{1}{\sqrt{1-\frac{2 m}{r}+\frac{q^{2}}{r^{2}}}}\right) \\
& a^{\lambda}=s\left(0,0,0, \frac{q}{m}\right) \\
& \rho_{c}=\frac{q^{3}}{m} \frac{\sqrt{1-\frac{2 m}{r}+\frac{q^{2}}{r^{2}}}}{r^{4}} \\
& \rho_{m}=\frac{q^{4}}{m^{2}} \frac{\left(1-\frac{2 m}{r}+\frac{q^{2}}{r^{2}}\right)}{r^{4}}
\end{aligned}
$$

In (44) I have introduced a parameter $s$, where $s= \pm 1$ corresponding to the global matter-to-antimatter transformation symmetry (30), which will be further discussed in section 5.4 The matter and antimatter solutions to the proposed theory that emerge here are a direct result of treating $\rho_{m}$ and $\rho_{c}$ as dynamic fields to be solved for which differs from the traditional classical treatment in which they are treated as external fields inserted into the theory. Except for the possibility of both matter and antimatter solutions, the physical interpretation of solution (44) is almost identical to that of the M\&EFEs, i.e., a non-rotating, spherically symmetric particle having charge $q$ and mass $m$. The metric tensor which is identical to the Reissner-Nordström metric establishes that the new theory predicts a gravitational field identical to the prediction of Einstein's General Relativity. However, solution (44) does differ from the classical picture of 
a point charge in that the mass and charge of the particle in (44) are not localized, with both $\rho_{m}$ and $\rho_{c}$ tailing off as $1 / r^{4}$.

Of note in solution (44) is the value of $a^{\lambda}$ with all of its spatial components being zero. This guarantees $a^{\lambda} R_{\lambda}{ }^{\nu}$ is time-like as required by the definition of charge density $\rho_{c}$ given in (12). Also of note is the form of the radial electric field,

$$
E_{r}=\frac{q}{r^{2}}-\frac{q^{3} / m}{r^{3}}=\frac{q}{r^{2}}\left(1-\frac{q^{2} / m}{r}\right)
$$

which agrees with the Coulomb field $q / r^{2}$ to leading order in $1 / r$. However, going beyond the leading order term, the next term in the radial electric field depends on both the charge and mass of the particle. Taking an electron as an example, its electric field as given by (45) would be,

$$
E_{r}=\frac{q_{e}}{r^{2}}\left(1-\frac{r_{e}}{r}\right) \approx \frac{q_{e}}{r^{2}}\left(1-\frac{2.82 \times 10^{-15} \mathrm{~m}}{r}\right)
$$

where $r_{e}=q_{e}^{2} / m_{e}$ is recognized as the classical radius of an electron $\left(\sim 2.82 \times 10^{-15} \mathrm{~m}\right)$. Although the correction term to the Coulomb field is small, being only $53 \mathrm{ppm}$ at a Bohr radius, it may have interesting consequences in various situations because it depends on both the charge and the mass of the particle.

As already mentioned, the new theory's solution (44) goes further than the M\&EFE's solution by solving for the spatial dependence of both the charge density $\rho_{c}$ and the mass density $\rho_{m}$, solutions which then define the spatial dependence of the energy-momentum tensor,

$$
T^{\mu v}=\rho_{m} u^{\mu} u^{v}+F_{\lambda}^{\mu} F^{v \lambda}-\frac{1}{4} g^{\mu v} F^{\rho \sigma} F_{\rho \sigma} .
$$

By direct substitution it can be verified that the resulting spatial dependence of the energy momentum tensor (47) along with the Reissner-Nordström metric (37) do not satisfy Einstein's equation of General Relativity (24). However, Einstein's equation of General Relativity augmented by the $\Lambda^{\mu v}$ term on the RHS of (28) is trivially satisfied. For completeness, the values of $G^{\mu v}, T^{\mu v}$ and $\Lambda^{\mu v}$ that go with solution (44) are given here: 


$$
\begin{aligned}
& G^{\mu \nu}=\left(\begin{array}{cccc}
\frac{q^{2}\left(q^{2}+r(-2 m+r)\right)}{r^{6}} & 0 & 0 & 0 \\
0 & -\frac{q^{2}}{r^{6}} & 0 & 0 \\
0 & 0 & -\frac{q^{2} \csc ^{2}(\theta)}{r^{6}} & 0 \\
0 & 0 & 0 & -\frac{q^{2}}{r^{2}\left(q^{2}+r(-2 m+r)\right)}
\end{array}\right)
\end{aligned}
$$

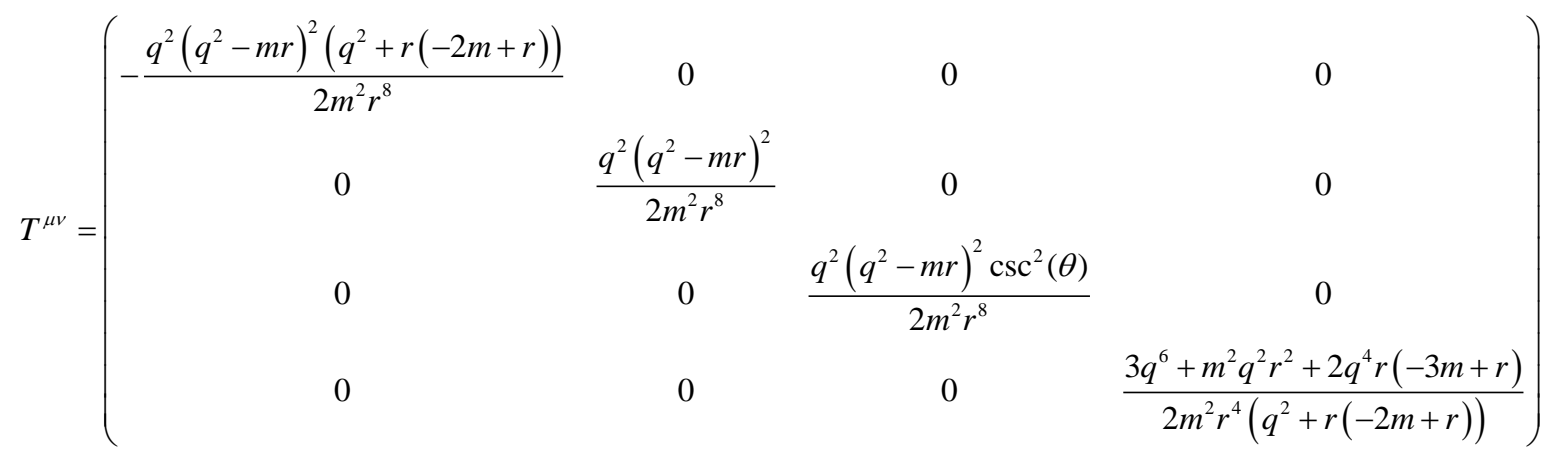

$$
\begin{aligned}
& \Lambda^{\mu v}=G^{\mu v}+8 \pi T^{\mu v}
\end{aligned}
$$

In the context of classical General Relativity (24), the interpretation of $\Lambda^{\mu v}$ is that of dark matter and/or dark energy, which serves as a source term for gravitational fields in addition $T^{\mu \nu}$. However, in the context of the proposed theory, $\Lambda^{\mu v}$ depends only on the existence of normal matter and normal energy and is a consequence of the fundamental field equations (1) through (3), again emphasizing that theory of gravitation emerging from the proposed theory differs from that of classical General Relativity as given by (24).

Another feature that results from the spatial distributions for both the charge and mass density being specified as part of the solution (44) is that it gives a mechanism for quantizing the mass $m$ and charge $q$, both parameters of the Reissner-Nordström metric (37). By requiring that both the mass $m$ and charge $q$ be consistent with the spatially integrated mass density and charge density, respectively, selfconsistency equations can be derived that put additional requirements on the physically allowable values these parameters can have, a topic that will be picked up in section 5.6. 
Finally, an interesting constraint on particle-like solutions with metrics that depend explicitly on a charge parameter $q$, such as the Reissner-Nordström metric used in (44) follows from the charge-conjugation symmetry (29). The charge conjugation symmetry transformation (29) takes $g_{\mu \nu} \rightarrow g_{\mu v}$ and $\rho_{c} \rightarrow-\rho_{c}$ , or equivalently $q \rightarrow-q$, as will be justified in section 5.6. This forces the conclusion that the sign of $q$ has no impact on the metric, i.e., the metric can only depend on the absolute value of $q$ (or the absolute value of $\rho_{c}$ ) since it is unchanged by the transformation $q \rightarrow-q$. This result, which is applicable to all solutions of fundamental field equations (1) through (3) is also in-line with known charge containing solutions of Einstein's field equation such as the Reissner-Nordström and Kerr-Newman metrics, both of which depend on $q^{2}$.

\subsection{Electromagnetic radiation}

Working in the weak field limit, I derive expressions for a propagating electromagnetic plane waves in terms of the vector field $a^{\lambda}$ and the metric tensor $g_{\mu v}$. This example is useful because it establishes a fundamental relationship between electromagnetic and gravitational radiation imposed by equation (1) and predicts that electromagnetic and gravitational waves are both manifestations of wave propagation of the underlying metric $g_{\mu \nu}$. To begin, consider an electromagnetic plane wave having frequency $\omega$, propagating in the $+z$-direction and polarized in the $x$-direction. The Maxwell tensor for this field is given by,

$$
F_{\mu \nu}=\left(\begin{array}{cccc}
0 & 0 & -B_{y} & E_{x} \\
0 & 0 & 0 & 0 \\
B_{y} & 0 & 0 & 0 \\
-E_{x} & 0 & 0 & 0
\end{array}\right) e^{i \omega(t-z)}
$$

where $E_{x}$ and $B_{y}$ are the constant field amplitudes of the electromagnetic wave. Next, assume a nearMinkowski weak field metric given by,

$$
\begin{gathered}
g_{\mu v}=\eta_{\mu v}+h_{\mu v} e^{i \omega(t-z)} \\
\left|h_{\mu v}\right| \ll 1
\end{gathered}
$$

where $\eta_{\mu \nu}=\operatorname{diag}[1,1,1,-1], h_{\mu \nu}$ are complex constants, and the vector field $a^{\lambda}$ is also assumed to be constant and given by, 


$$
a^{\lambda}=\left(a^{1}, a^{2}, a^{3}, a^{4}\right)
$$

I proceed by substituting for $F_{\mu \nu}$ from (49), $g_{\mu \nu}$ from (50), and $a^{\lambda}$ from (51) into (1), and then only retain terms to first order in the fields $h_{\mu \nu}$ and $F_{\mu \nu}$, both of which are assumed to be small and of the same order $^{[15]}$ Doing this leads to a set of 8 independent linear equations for the 16 unknown constants: $h_{\mu v}$, $a^{\lambda}, E_{x}$ and $B_{y}$. Solving these 8 independent equations, the 8 field components $E_{x}, B_{y}, h_{13}, h_{22}, h_{23}$ , $h_{34}, a^{2}$ and $a^{3}$ can be solved for in terms of 8 free constants $a^{1}, a^{4}, h_{11}, h_{12}, h_{14}, h_{24}, h_{33}$, and $h_{44}$ :

$$
\begin{gathered}
E_{x}=i \omega \frac{\left(h_{11}{ }^{2}+h_{12}{ }^{2}\right)}{2 h_{11}} a^{1}, \\
B_{y}=E_{x} \\
g_{\mu \nu}=\eta_{\mu \nu}+\left(\begin{array}{cccc}
h_{11} & h_{12} & -h_{14} & h_{14} \\
h_{12} & -h_{11} & -h_{24} & h_{24} \\
-h_{14} & -h_{24} & h_{33} & -\frac{1}{2}\left(h_{33}+h_{44}\right) \\
h_{14} & h_{24} & -\frac{1}{2}\left(h_{33}+h_{44}\right) & h_{44}
\end{array}\right) e^{i \omega(t-z)},
\end{gathered}
$$

and

$$
a^{\lambda}=\left(a^{1}, a^{1} \frac{h_{12}}{h_{11}}, a^{4}, a^{4}\right)
$$

This solution illustrates several ways in which the new theory departs from the traditional view of electromagnetic radiation. Of most significance, the undulations in the electromagnetic field are due to underlying undulations in the metric field $g_{\mu v}$ (53) via the coupling defined in (1). This result also underscores that the existence of electromagnetic radiation is forbidden in strictly flat space-time. An interesting aspect of this solution is that while electromagnetic radiation necessitates the presence of an underlying gravitational radiation field, the underlying gravitational radiation is not completely defined by the electromagnetic radiation. The supporting gravitational radiation has 6 undetermined constants $\left(h_{11}, h_{12}, h_{14}, h_{24}, h_{33}, h_{44}\right)$ with the only restriction being $\left|h_{\mu v}\right| \ll 1$ and $h_{11} \neq 0$ as required by (52). 
Further insight into the physical content of the metric (53) is evident after making the infinitesimal coordinate transformation from $x^{\mu} \rightarrow x^{\prime \mu}$ given by,

$$
\left(\begin{array}{c}
x \\
y \\
z \\
t
\end{array}\right) \rightarrow\left(\begin{array}{c}
x^{\prime} \\
y^{\prime} \\
z^{\prime} \\
t^{\prime}
\end{array}\right)=\left(\begin{array}{c}
x+\frac{i}{\omega} h_{14} e^{i \omega(t-z)} \\
y+\frac{i}{\omega} h_{24} e^{i \omega(t-z)} \\
z-\frac{i}{2 \omega} h_{33} e^{i \omega(t-z)} \\
t-\frac{i}{2 \omega} h_{44} e^{i \omega(t-z)}
\end{array}\right)
$$

and only retaining terms to first order in the $h$ 's. Doing this, the metric (53) is transformed to,

$$
g_{\mu \nu}^{\prime}=\eta_{\mu \nu}+\left(\begin{array}{cccc}
h_{11} & h_{12} & 0 & 0 \\
h_{12} & -h_{11} & 0 & 0 \\
0 & 0 & 0 & 0 \\
0 & 0 & 0 & 0
\end{array}\right) e^{i \omega(t-z)}
$$

while $E^{\prime}{ }_{x}$ and $B^{\prime}{ }_{y}$, the transformed electric and magnetic field amplitudes, respectively, are identical to $E_{x}$ and $B_{y}$ given in (52). Note, only the $h_{11}$ and $h_{12}$ components of the metric (56) have an absolute physical significance, and $h_{22}=-h_{11}$ which makes the plane wave solution (56) identical to the gravitational plane wave solution of the classical Einstein field equations. ${ }^{[16], ~[17] ~}$

Because the underlying gravitational wave couples to both charged and uncharged matter, one consequence of the solution here is that there will be an uncertainty when describing the interaction of electromagnetic radiation with matter if the gravitational wave component of the problem is ignored. However, for nonrelativistic matter, this gravitational interaction (56) vanishes to first order in the $h$ 's. To see this, consider the following expansion of the Lorentz force law,

$$
\begin{gathered}
\rho_{m} \frac{D u^{\mu}}{D \tau}=\rho_{c} u^{\lambda} F_{\lambda}^{\mu} \\
\downarrow \\
\rho_{m} \frac{d u^{\mu}}{d \tau}=-\rho_{m} u^{v} u^{\lambda} \Gamma_{\nu \lambda}^{\mu}+\rho_{c} u^{\lambda} F_{\lambda}^{u}
\end{gathered}
$$


The first term on the RHS in the line above represents the gravitational interaction. This gravitational interaction term vanishes for nonrelativistic matter with $u^{\lambda} \approx(0,0,0,1)$ because for the metric (56) all the $\Gamma_{44}^{\mu}$ vanish to first order in the $h^{\prime} s$.

\subsection{Gravitational radiation}

The forgoing analysis demonstrates the necessity of having an underlying gravitational wave to support the presence of an electromagnetic wave, but the converse is not true and gravitational radiation can exist independent of any electromagnetic radiation. The following analysis demonstrates this by solving for the structure of gravitational radiation in the absence of electromagnetic radiation. Following the same weak field formalism for the unknown fields $h_{\mu \nu}$ given in (50), but this time zeroing out $E_{x}$ and $B_{y}$ in (49), leads to the following solutions for $g_{\mu \nu}$ and $a^{\lambda}$,

$$
g_{\mu \nu}=\eta_{\mu \nu}+\left(\begin{array}{cccc}
h_{11} & h_{12} & -h_{14} & h_{14} \\
h_{12} & \frac{h_{12}^{2}}{h_{11}} & -h_{24} & h_{24} \\
-h_{14} & -h_{24} & h_{33} & -\frac{h_{33}+h_{44}}{2} \\
h_{14} & h_{24} & -\frac{h_{33}+h_{44}}{2} & h_{44}
\end{array}\right) e^{i \omega(t-z)}
$$

and

$$
a^{\lambda}=\left(a^{1},-a^{1} \frac{h_{11}}{h_{12}}, a^{4}, a^{4}\right)
$$

Both $g_{\mu v}$ given by (58) and $a^{\lambda}$ given by (59) are modified from their solutions in the presence of an electromagnetic wave as given by (53) and (54), respectively. Performing a transformation to the same primed coordinate system as given in (55), here gives the metric field,

$$
g_{\mu \nu}^{\prime}=\eta_{\mu \nu}+\left(\begin{array}{cccc}
h_{11} & h_{12} & 0 & 0 \\
h_{12} & \frac{h_{12}^{2}}{h_{11}} & 0 & 0 \\
0 & 0 & 0 & 0 \\
0 & 0 & 0 & 0
\end{array}\right) e^{i \omega(t-z)}
$$


illustrating again that only the $h_{11}$ and $h_{12}$ components have an absolute physical significance. The interaction of nonrelativistic matter with the gravitational wave (60) vanishes to first order in the $h^{\prime} s$ for the same reason that it vanished for the gravitational wave (56) that accompanies electromagnetic radiation. Of particular note is the change in the value of the $h_{22}$ component depending on whether the gravitational wave supports an electromagnetic wave as in (56) or is standalone as in (60).

\subsection{Isotropic and homogeneous universe}

As shown in a section 4.1, the M\&EFEs and fundamental field equations (1) through (3) share particle-like solutions having a similar character. However, when considering non-static metrics, differences between the predictions of the two theories start to emerge. To illustrate some of these differences, here I investigate the Friedmann-Lemaître-Robertson-Walker (FLRW) metric,

$$
g_{\mu v}=\left(\begin{array}{cccc}
\frac{R_{c s}{ }^{2}(t)}{1-k r^{2}} & 0 & 0 & 0 \\
0 & R_{c s}{ }^{2}(t) r^{2} & 0 & 0 \\
0 & 0 & R_{c s}{ }^{2}(t) r^{2} \operatorname{Sin}^{2}(\theta) & 0 \\
0 & 0 & 0 & -1
\end{array}\right)
$$

where $k$ equals $+1,0$ or -1 depending on whether the spatial curvature is positive, zero or negative, respectively, and $R_{c s}(t)$ is the cosmic scale factor at time $t$. Just as in the case of Einstein's equation of General Relativity where the FLRW metric is a cosmological solution representing a homogeneous and isotropic universe, it is the same for fundamental field equations (1) through (3) with an appropriate choice for the time development of the cosmic scale parameter $R_{c s}(t)$. To derive the time dependence of this cosmic scale factor I start by noting that the 3-dimensional spatial subspace of (61) is maximally symmetric and so any tensor fields that inhabit that subspace must also be maximally symmetric. ${ }^{[18]}$ Specifically, this restricts the form of $a^{\mu}$ to be,

$$
a^{\mu}=\left(0,0,0, a^{4}(t)\right)
$$

and forces the antisymmetric Maxwell tensor to vanish,

$$
F_{\mu v}=0
$$

Because $F_{\mu v}$ vanishes so must $F_{\mu v ; \kappa}$, 


$$
F_{\mu v ; \kappa}=0,
$$

which on substitution in (1) forces,

$$
a^{\lambda} R_{\lambda \kappa \mu \nu}=0
$$

This in turn forces,

$$
a^{\lambda} R_{\lambda}^{v}=0
$$

which is just equation (12) with $\rho_{c}=0$. Substituting $a^{\mu}$ given by (62), and the FLRW metric given by (61) into (65) then leads to the following set of equations to be satisfied,

$$
\begin{gathered}
a^{4}(t) R_{4114}=a^{4}(t)\left(\frac{R_{c s}(t)}{k r^{2}-1} \frac{d^{2} R_{c s}(t)}{d t^{2}}\right)=0 \\
a^{4}(t) R_{4224}=a^{4}(t)\left(-r^{2} R_{c s}(t) \frac{d^{2} R_{c s}(t)}{d t^{2}}\right)=0 \\
a^{4}(t) R_{4334}=a^{4}(t)\left(-r^{2} R_{c s}(t) \operatorname{Sin}^{2}(\theta) \frac{d^{2} R_{c s}(t)}{d t^{2}}\right)=0
\end{gathered}
$$

with all other components of (65) not listed in (67) being trivially satisfied, i.e., $0=0$. The nontrivial component equations (67) are all satisfied if,

$$
\frac{d^{2} R_{c s}(t)}{d t^{2}}=0
$$

which gives,

$$
R_{c s}(t)=R_{c s 0}+v_{c s} t
$$

where $R_{c s 0}$ is the cosmic scale factor at time $t=0$ and $v_{c s}$ is the rate of change of the cosmic scale factor. The solution for $R_{c s}(t)$ given in (69) ensures that the metric (61) satisfies both (65) and (66) for all values of $k$. Based on this solution, the predictions of the new theory for a homogeneous and isotropic universe are:

1. It must be charge neutral, i.e., $\rho_{c}=0$.

2. The cosmic scale factor changes linearly with cosmic time. 
3. The spatial curvature of the universe can be positive, negative or 0 .

The second prediction above runs counter to results of the Friedmann models of classical General Relativity in which the growth of the cosmic scale factor is divided into three regimes: the radiation dominated regime with the scale factor growing as $t^{1 / 2}$, the matter dominated regime with the scale factor growing as $t^{2 / 3}$, and the dark energy dominated regime with the scale factor growing exponentially with time. This emphasizes one of the challenges facing the proposed theory based on fundamental equations (1) through (3), that of finding additional solutions that are in agreement with the interpretation of recent observations and analyses indicating an accelerating universe.

Just as in the case of the spherically symmetric particle-like solution analyzed in section 4.1, the cosmological solution of fundamental field equations (1) through (3) analyzed here must satisfy Einstein's equation of General Relativity augmented by a $\Lambda^{\mu v}$ term on its RHS (28). For the cosmological solution considered here it is straight forward to calculate the $G^{\mu v}, T^{\mu v}$ and $\Lambda^{\mu v}$ by taking the energymomentum tensor to be,

$$
T^{\mu v}=\left(p+\rho_{m}\right) u^{\mu} u^{v}+p g^{\mu v}
$$

and the four-velocity vector field to be,

$$
u^{\mu}=(0,0,0,1)
$$

where the form of the energy-momentum tensor (70) as a perfect fluid with pressure $p$ and mass-density $\rho_{m}$, and the form of the four-velocity $u^{\mu}$ in (71) are both dictated by the requirement that they be maximally symmetric in the 3-dimensional spatial subspace of (61). For completeness the values of $G^{\mu v}, T^{\mu \nu}$ and $\Lambda^{\mu v}$ that go with (69), (70) and (71) are given here: 


$$
\begin{aligned}
& G^{\mu v}=\left(\begin{array}{cccc}
\frac{k-k^{2} r^{2}+v_{C S}{ }^{2}-k r^{2} v_{C S}{ }^{2}}{\left(R_{C S 0}+t v_{C S}\right)^{4}} & 0 & 0 & 0 \\
0 & \frac{k+v_{C S}{ }^{2}}{r^{2}\left(R_{C S 0}+t v_{C S}\right)^{4}} & 0 & 0 \\
0 & 0 & \frac{\left(k+v_{C S}{ }^{2}\right) \csc ^{2}(\theta)}{r^{2}\left(R_{C S 0}+t v_{C S}\right)^{4}} & 0 \\
0 & 0 & 0 & -\frac{3\left(k+v_{C S}{ }^{2}\right)}{\left(R_{C S 0}+t v_{C S}\right)^{2}}
\end{array}\right) \\
& T^{\mu \nu}=\left(\begin{array}{cccc}
\frac{\left(1-k r^{2}\right) p(t)}{\left(R_{C S 0}+t v_{C S}\right)^{2}} & 0 & 0 & 0 \\
0 & \frac{p(t)}{r^{2}\left(R_{C S 0}+t v_{C S}\right)^{2}} & 0 & 0 \\
0 & 0 & \frac{\csc ^{2}(\theta) p(t)}{r^{2}\left(R_{C S 0}+t v_{C S}\right)^{2}} & 0 \\
0 & 0 & 0 & \rho_{m}(t)
\end{array}\right) \\
& \Lambda^{\mu v}=G^{\mu v}+8 \pi T^{\mu v}
\end{aligned}
$$

Just as was the case for the spherically symmetric particle-like solution studied in section 4.1, in the context of classical General Relativity (24) the interpretation of $\Lambda^{\mu v}$ is that of dark matter and/or dark energy. However, in the context of the new theory, $\Lambda^{\mu v}$ depends only on the existence of normal matter and normal energy as determined by fundamental field equations (1) through (3).

\section{DISCUSSION}

\subsection{The classical Maxwell's field equations from $\boldsymbol{F}_{\mu v ; \kappa}=a^{\lambda} \boldsymbol{R}_{\lambda \kappa \mu v}$}

It is an unusual circumstance that equation (1) contains both $a^{\lambda}$ and the R-C tensor explicitly, but the classical Maxwell equations which are derivative to (1) contain neither an explicit reference to $a^{\lambda}$ or the R-C tensor,

$$
F_{\mu \nu ; \kappa}=a^{\lambda} R_{\lambda \kappa \mu \nu} \rightarrow\left\{\begin{array}{c}
F_{\mu \nu, \kappa}+F_{v \kappa, \mu}+F_{\kappa \mu, v}=0 \\
F_{; \mu}^{\mu \nu}=-\rho_{c} u^{v}
\end{array}\right.
$$


This circumstance gives the classical Maxwell equations the appearance of being valid in flat space-time and motivates their classical implementation. However, in the view of the proposed theory this apparent validity of the classical Maxwell equations in flat space-time is due the conserved charge density $\rho_{c}$, which is classically assumed to exist in flat space-time but in the proposed theory is explicitly tied to curved space-time through the identification (12). Thus, the classical Maxwell equations in flat space-time are at best an approximation to the theory of electromagnetism that follows from fundamental field equations (1) through (3) and which require curved space-time for all electromagnetic phenomena.

\subsection{Dark matter and dark energy}

With General Relativity as the foundation of observational gravitational physics today, dark matter and dark energy have been postulated to exist because of the many galactic and cosmological scale observations that cannot be understood using General Relativity with normal matter and normal energy alone. Specifically, some of the large-scale gravitational features of galaxies and galactic clusters dating back to Zwicky's observations in the 1930's have been explained using dark matter ${ }^{[19]}$, and the acceleration of the universe discovered in the 1990's has been explained using dark energy ${ }^{[20]}$. Another example of modifications made to the original General Relativity field equations (24) to satisfy a perceived need was the cosmological constant term $\lambda g_{\mu \nu}$ that Einstein added to their RHS,

$$
G_{\mu \nu}=-8 \pi T_{\mu \nu}+\lambda g_{\mu \nu}
$$

Specifically, this was done to enable a static universe solution but then subsequently dropped after expansion was discovered. Today this term is again in vogue as a possible representation of dark energy.

One of the vexing problems facing dark matter and dark energy-based explanations of various observational phenomena today is an ongoing inability to directly detect these forms of matter and energy, a situation which only adds to their ad hoc character. However, fundamental field equations (1) through (3) offer the prospect that dark matter and dark energy effects can be explained in terms of normal matter and normal energy alone, i.e., the $\Lambda^{\mu v}$ term in (28) representing dark matter and dark energy in the context of General Relativity is provided with a mechanism for directly calculating its structure using fundamental field equations (1) through (3) and only normal matter and normal energy. The already investigated spherically symmetric particle-like solution which assumed a ReissnerNordström metric and the cosmological solution which assumed an FLRW metric are two accessible examples that outline such a direct calculation of $\Lambda^{\mu v}$. With questions today regarding the validity of 
classical General Relativity beyond the confines of our own solar system ${ }^{[21]}$ and the inability to directly detect dark matter and dark energy, the possible interpretation of the $\Lambda^{\mu v}$ term in (28) using only normal matter and normal energy is an enticing feature of fundamental field equations (1) through (3). However, it must be acknowledged that one of the challenging tasks facing the proposed field theory, and one well beyond the analysis presented in this manuscript, is that of finding additional solutions that could be interpreted as agreeing with the rapidly developing observational understanding of galactic and cosmological structures.

\subsection{The unification of gravitational and electromagnetic radiation}

One of the successes of fundamental field equation (1) is the existence of solutions describing both electromagnetic and gravitational radiation, with both phenomena being unified as undulations of the underlying metric field $g_{\mu \nu}$. Because both gravitational and electromagnetic radiation are due to

undulations of the metric field $g_{\mu \nu}$, their speed of propagation is predicted to be identical in the proposed theory. This prediction has recently been refined experimentally with observations made during the binary neutron star merger in NGC 4993, 130 million light years from Earth. ${ }^{[22]}$ The nearly simultaneous detection, within 2 seconds of each other, of gravity waves ${ }^{[23]}$ and a burst of gamma rays ${ }^{[24]}$ from this event experimentally constrain the propagation speed of electromagnetic and gravitational radiation to be the same to better than 1 part in $10^{15}$.

\subsection{The emergence of antimatter and its behavior in electromagnetic and gravitational fields}

One of the unique features of fundamental field equations (1) through (3) as a classical field theory is that the properties of matter and antimatter emerge naturally in solutions. Traditionally, these properties emerge in quantum mechanical treatments but here emerge in the context of a classical continuous field theory due to the global symmetry (30) of the fundamental equations (1) through (3). This feature of solutions to the fundamental field equations is intimately tied to the treatment of $\rho_{c}$ and $\rho_{m}$ as dynamic fields that are solved for rather than being externally inserted as done in the traditional classical physics picture. For example, every matter containing solution to equations (1) through (3) has a corresponding antimatter solution generated by the symmetry transformation (30). This is evident in the spherically symmetric particle-like solution (44) where the multiplicative factor $s$ in the expressions for $F_{\mu \nu}, a^{\lambda}$ and $u^{\lambda}$ is defined by, 


$$
s=\left\{\begin{array}{l}
+1 \text { for matter } \\
-1 \text { for antimatter }
\end{array}\right.
$$

and accounts for the matter-antimatter symmetry expressed in (30). The physical interpretation of the $s=-1$ solution is that it represents a particle having the same mass but opposite charge and four-velocity to that of the $s=+1$ solution. This is equivalent to the view that a particle's antiparticle is the particle moving backwards through time. ${ }^{[25]}$ Said another way, the time-like component of the four-velocity is positive for matter and negative for antimatter,

$$
u^{4}\left\{\begin{array}{l}
>0 \text { for matter } \\
<0 \text { for antimatter }
\end{array}\right.
$$

With these definitions for the four-velocity of matter and antimatter, charged mass density can annihilate similarly charged anti-mass density and satisfy both the local conservation of charge (18) and local conservation of mass (22). Additionally, such annihilation reactions must conserve energy by (3).

Building on the distinction between matter and antimatter, their behavior in external electromagnetic and gravitational fields in the context of the theory based on fundamental field equations (1) through (3) is briefly reviewed here. As already mentioned, antimatter can be viewed as matter moving backwards through time. To see this more rigorously consider the four-velocity associated with a fixed quantity of charge and mass density,

$$
u^{\lambda}=\frac{d x^{\lambda}}{d \tau}
$$

Under the matter-antimatter transformation (30), $u^{\lambda} \rightarrow-u^{\lambda}$, or equivalently $d \tau \rightarrow-d \tau$. This motivates the following expression for the four-velocity in terms of the coordinate time in locally inertial coordinate systems,

$$
u^{\lambda}=\frac{d x^{\lambda}}{d \tau}=s \gamma \frac{d x^{\lambda}}{d t}=s \gamma\left(\begin{array}{l}
\vec{v} \\
1
\end{array}\right)
$$

where $s$ is the matter-antimatter parameter defined in (75), $\vec{v}$ is the ordinary 3-space velocity of the charge and mass density, and $\gamma=1 / \sqrt{1-\vec{v}^{2}}$. Equation (78) establishes that corresponding matter and antimatter solutions travel in opposite time directions relative to each other. One of the unusual aspects 
of the matter-antimatter transformation (30) is that $\rho_{c}$ does not change sign under the transformation. To see that this is consistent with the usual view in which antiparticles have the opposite charge of their corresponding particles, I use (78) to illustrate the behavior of a charged matter and antimatter density in an external electromagnetic field. Consider a region with an externally defined electromagnetic field,

$$
F_{\mu \nu}=\left(\begin{array}{cccc}
0 & B_{z} & -B_{y} & E_{x} \\
-B_{z} & 0 & B_{x} & E_{y} \\
B_{y} & -B_{x} & 0 & E_{z} \\
-E_{x} & -E_{y} & -E_{z} & 0
\end{array}\right)
$$

in a locally inertial coordinate system. Starting with the Lorentz force law (23), and then expanding and rearranging slightly leads to the following development,

$$
\begin{gathered}
\rho_{p} \frac{D u^{\mu}}{D \tau}=\rho_{c} F_{\lambda}^{\mu} u^{\lambda} \\
\downarrow \\
\rho_{p} s \gamma \frac{d u^{\mu}}{d t}=\rho_{c} F_{\lambda}^{\mu} u^{\lambda} \\
\downarrow \\
\rho_{p} s \gamma \frac{d}{d t}\left(\begin{array}{c}
s \gamma \vec{v} \\
s \gamma
\end{array}\right)=\rho_{c}\left(\begin{array}{cccc}
0 & B_{z} & -B_{y} & E_{x} \\
-B_{z} & 0 & B_{x} & E_{y} \\
B_{y} & -B_{x} & 0 & E_{z} \\
E_{x} & E_{y} & E_{z} & 0
\end{array}\right)\left(\begin{array}{l}
s \gamma v_{x} \\
s \gamma v_{y} \\
s \gamma v_{z} \\
s \gamma
\end{array}\right) \\
\downarrow
\end{gathered}
$$

which on the last line above ends up at the conventional form of the Lorentz force law except for the extra factor of $s$ on the RHS. This factor of $s$ in (80) gives the product $s \rho_{c}$ the appearance that antimatter charge density has the opposite sign to that of matter charge density when interacting with an external electromagnetic field.

Next, I investigate the behavior of antimatter in an external gravitational field. There is no question about the gravitational fields generated by matter and antimatter, they are identical under the matterantimatter symmetry (30) as $g_{\mu \nu}$ is unchanged by that transformation. To understand whether 
antimatter is attracted or repelled by an external gravitational field, I again go to the Lorentz force law (23) but this time assume there is no electromagnetic field present, just a gravitational field given by a Schwarzschild metric generated by a central mass $m>0$ that is composed of either matter or antimatter. I explicitly call out $m>0$ because I am endeavoring to develop a physical theory that axiomatically flows from fundamental field equations (1) through (3) and at this point in the development there is nothing to preclude the existence of negative mass density $\rho_{m}<0$, a consideration I will return to in section 5.5. Placing a test particle having mass $m_{\text {test }}$ composed of either matter or antimatter a distance $r$ from the center of the gravitational field and assuming it to be initially at rest, the trajectory of the test particle is that of a geodesic given by the following development,

$$
\begin{gathered}
m_{\text {test }} \frac{D u^{\mu}}{D \tau}=0 \\
\downarrow \\
s \gamma \frac{d u^{\mu}}{d t}=-\Gamma_{\nu \rho}^{\mu} u^{v} u^{\rho} \\
\downarrow \\
s \gamma \frac{d}{d t}\left(s \gamma \frac{d}{d t}\left(\begin{array}{l}
r \\
\theta \\
t
\end{array}\right)\right)=-\Gamma_{v \rho}^{\mu} u^{v} u^{\rho} \approx-\Gamma_{44}^{\mu} u^{4} u^{4}=-\left(\begin{array}{c}
\left.1-\frac{2 m}{r}\right) \frac{m}{r^{2}} \\
0 \\
0 \\
0
\end{array}\right)\left(\begin{array}{c}
\left.\frac{m}{\sqrt{1-\frac{2 m}{r}}}\right)^{2} \\
\frac{r^{2}}{0} \\
0 \\
0
\end{array}\right) s^{2}
\end{gathered}
$$

where $s= \pm 1$ references whether the test particle is composed of matter or antimatter (75). In the last line of (81) I have approximated the RHS using the initial at rest value of the test particle's four-velocity $u^{\mu}=(0,0,0, s / \sqrt{1-2 m / r})$, and additionally used the fact that the only nonzero $\Gamma_{44}^{\mu}$ in a Schwarzschild metric is $\Gamma_{44}^{1}=\left(1-\frac{2 m}{r}\right) m / r^{2}$. Simplifying the LHS of the last line in (81) by noting that initially $\gamma=1$ then gives,

$$
\frac{d^{2} r}{d t^{2}} \approx-\frac{m}{r^{2}}
$$

which is independent of $s$, and so demonstrates that the proposed theory predicts both matter and antimatter test particles will be attracted by the source of the gravitational field, and this regardless of 
whether the source of the gravitational field is matter or antimatter. The result that the test particle is attracted toward the source of the gravitational field is also independent of whether the test particle's mass, $m_{\text {test }}$, is positive or negative, this because the geodesic trajectory (82) is independent of $m_{\text {test }}$.

\subsection{Possibility of negative mass solutions and antigravity}

As already noted, there appears to be nothing in the fundamental equations (1) through (3) that precludes the possibility of negative mass density $\rho_{m}<0$. The existence of negative mass density is equivalent to the existence of antigravity because negative mass density generates gravitational fields that are repulsive, viz., equation (82) with $m<0$. However, logical inconsistencies are introduced if negative mass density were to exist. As just shown, equation (82) with $m>0$ predicts a test particle at some distance from the origin will feel an attractive gravitational force regardless of whether the test particle is comprised of matter or antimatter and regardless of whether its mass is positive or negative. Now consider equation (82) with the central mass $m<0$. Using the same argument as in the previous section, the test particle in this case will feel a repulsive gravitational force regardless of whether it is comprised of matter or antimatter and regardless of whether its mass is positive or negative. These two situations directly contradict each other. For example, in the first case the negative mass test particle is gravitationally attracted toward the positive mass particle located at the origin, but in the second case the positive mass test particle is gravitationally repelled by the negative mass particle located at the origin. This contradiction makes fundamental equations (1) through (3) logically inconsistent if negative mass density were to exist. The only way to avoid this logical contradiction is to require that mass density be non-negative always. This condition that mass density $\rho_{m}$ be non-negative always is also consistent with the global symmetry transformations (29) through (31) where it was noted that the field $\rho_{m}$ does not change sign under any of the symmetry transformations.

It is interesting to note that the existence of negative mass in the context of classical General Relativity has been extensively studied ${ }^{[26], ~[27] ~ a n d ~ i n v o k e d, ~ p a r t i c u l a r l y ~ w h e n ~ t r y i n g ~ t o ~ f i n d ~ s t a b l e ~ p a r t i c l e-l i k e ~}$ solutions using the conventional Einstein field equations. ${ }^{[28], ~[29], ~[30] ~ H o w e v e r, ~ i n ~ t h e ~ c o n t e x t ~ o f ~ t h e ~ p r e s e n t ~}$ theory the existence of negative mass density leads to a logical contradiction that can only be resolved by requiring mass density be non-negative always, i.e., $\rho_{m} \geq 0$. 
LLNL-JRNL-726138

\subsection{Conjecture for quantizing the charge and mass of particle-like solutions}

Consider particle-like solutions such as (44). Because the mass density and charge density are specified as part of the solution of fundamental field equations (1) through (3), a self-consistency constraint exists on physically allowed solutions that provides a mechanism for quantizing the charge and mass of such solutions. For example, in solution (44) the particle's total charge $q$ and total mass $m$, both parameters of the Reissner-Nordström metric, must agree with the spatially integrated charge and mass density, respectively, if the solution is to be self-consistent. For the charge, this amounts to requiring the asymptotic value of the electric field be consistent with the spatially integrated charge density,

$$
\lim _{r \rightarrow \infty} r^{2} F_{14}=\int \rho_{c} u^{4} \sqrt{\gamma_{s p}} d^{3} x=q
$$

where $q$ is the total charge of the particle and given by the asymptotic value of $r^{2} F_{14}$ per the solution given in (44), and $\gamma_{s p}$ is the determinant of the spatial metric defined by, ${ }^{[31]}$

$$
\gamma_{s p i j}=g_{i j}-\frac{g_{i 4} g_{j 4}}{g_{44}},
$$

where $i$ and $j$ run over the spatial dimensions 1, 2 and 3. An analogous quantizing boundary condition for the mass of the particle is arrived at by requiring the asymptotic value of its gravitational field be consistent with the spatially integrated mass density of the solution,

$$
\lim _{r \rightarrow \infty} r \frac{1+g_{44}}{2}=\int \rho_{m}\left|u^{4}\right| \sqrt{\gamma_{s p}} d^{3} x=m
$$

The reason for the absolute value of $u^{4}$ in the mass boundary condition (85) but not in the charge boundary condition (83) are the global symmetries (29) through (31) exhibited by the theory's fundamental field equations (1) through (3) and the requirement that the boundary conditions exhibit those same symmetries. The conjecture being put forth here is that boundary conditions (83) and (85) represent self-consistency constraints on the charge and the mass, respectively, that any particle-like solution to the fundamental field equations (1) through (3) must satisfy if the solutions are to be physically realizable.

For the spherically-symmetric solution investigated in (44), the RHS of both (83) and (85) diverge leaving no hope for satisfying these quantization/boundary conditions. The upshot of this observation is that while (44) represents a mathematical solution that describes the gravitational and electrical fields of a 
particle-like solution formally satisfying the fundamental field equations (1) through (3), (44) cannot represent a physically allowed solution. The possibility of finding solutions that satisfy both the fundamental field equations (1) through (3) and the charge and mass boundary conditions (83) and (85) remains an open question at this point. However, interesting possibilities exist beyond the spherically symmetric solution based on the Reissner-Nordström metric investigated within (44). For example, the modified Reissner-Nordström and modified Kerr-Newman metrics developed by S.M. Blinder ${ }^{[32]}$ give finite values for the RHS of both (83) and (85). Finally, when considering metrics that include nonzero angular momentum, as for example would be required for particles having an intrinsic magnetic field, the same approach used here to quantize the particle's mass and charge could be used to quantize its angular momentum. Traditionally the quantization of mass, charge and angular momentum are introduced in quantum mechanical treatments but here are conjectured within the framework of a classical continuous field-theoretic description of nature and are another example of how the proposed theory differs from the classical M\&EFEs.

\subsection{Possibility of superluminal transport if $a^{\lambda} R_{\lambda}{ }^{v}$ is space-like}

The form of the fundamental field equations given in Table I was strongly influenced by the desire to preserve as much as possible the physics embodied in the classical M\&EFEs, while simultaneously uniting them in a geometric framework under the assumption that the geometry of nature is Riemannian with four dimensions. This is the reason for the assumption that $a^{\lambda} R_{\lambda}{ }^{v}$ is time-like, which has been made through most of this manuscript. This assumption allows the identification of $\rho_{c}$ as a conserved quantity with its motion described by $u^{\nu}$, the same four-velocity used to describe the motion of the mass density $\rho_{m}$, and thus keeping the description of $\rho_{c}$ in the proposed theory the same as it is in the classical M\&EFEs. However, because I am attempting to develop the theory that flows axiomatically from fundamental equations (1) through (3), and because there is nothing a priori that requires that $a^{\lambda} R_{\lambda}{ }^{v}$ be time-like, for the remainder of this section I assume $a^{\lambda} R_{\lambda}{ }^{v}$ is space-like,

$$
\left(a^{\lambda} R_{\lambda}^{v}\right)\left(a^{\sigma} R_{\sigma \nu}\right)>0
$$

and investigate the consequences thereof. If $a^{\lambda} R_{\lambda}{ }^{v}$ is space-like, then the identification made in (12) in which $a^{\lambda} R_{\lambda}{ }^{v}$ was identified with the time-like quantity $\rho_{c} u^{v}$ is no longer valid. However, the 
fundamental field equations of the proposed theory as written in Table I remain valid and $a^{\lambda} R_{\lambda}{ }^{v}$ is still a conserved quantity as forced by equation (10) and the antisymmetry of $F_{\mu \nu}$,

$$
\left(a^{\lambda} R_{\lambda}^{v}\right)_{; \nu}=\frac{1}{\sqrt{g}} \frac{\partial}{\partial x^{v}}\left(\sqrt{g} a^{\lambda} R_{\lambda}^{v}\right)=0
$$

where $g=-\operatorname{Det}\left(g_{\mu \nu}\right)$. To understand implications of (87) more clearly when $a^{\lambda} R_{\lambda}{ }^{v}$ is space-like, I rewrite it in a locally inertial coordinate system that makes evident what the conserved quantity is, and at what speed the conserved quantity is transported,

$$
\frac{\partial}{\partial x^{i}}\left(a^{\sigma} R_{\sigma}{ }^{4} \frac{a^{\lambda} R_{\lambda}{ }^{i}}{a^{\kappa} R_{\kappa}{ }^{4}}\right)+\frac{\partial}{\partial t}\left(a^{\lambda} R_{\lambda}{ }^{4}\right)=0
$$

where i runs over the spatial indices 1-3 and 4 is the time index. Equation (88) represents the conservation of the quantity $a^{\lambda} R_{\lambda}{ }^{4}$ that is transported with ordinary 3-space velocity,

$$
v^{i}=\frac{a^{\lambda} R_{\lambda}{ }^{i}}{a^{\kappa} R_{\kappa}{ }^{4}} .
$$

The surprising feature of this 3-space velocity is that it is superluminal, with a magnitude greater than unity,

$$
|\vec{v}|>1
$$

due to $a^{\lambda} R_{\lambda}{ }^{v}$ being assumed space-like. At this point it is not possible to say much more. The mathematical implications of (86) and (87) taken together impose conservation in a local sense, but without specific solutions to fundamental equations (1) through (3) in which $a^{\lambda} R_{\lambda}{ }^{v}$ is space-like, one cannot say what the physical interpretation of the quantity being conserved by equation (87) is. Lacking specific solutions to the fundamental field equations in which $a^{\lambda} R_{\lambda}{ }^{v}$ is space-like, the prospect of superluminal transport must be considered speculative at this point. However, the possibility of superluminal transport in the context of a classical field theory may be an interesting and timely avenue of investigation as recent research may suggest the existence of nonlocal correlations stronger than those predicted by quantum theory. ${ }^{[33]}$ 
LLNL-JRNL-726138

\section{CONCLUSION}

The purpose of this manuscript was to review, correct and extend a recently proposed classical field theory in which the classical Maxwell field equations are replaced by equation (1) which couples the Maxwell tensor to the Riemann-Christoffel curvature tensor in a fundamentally new way, and to then demonstrate how this theory puts electromagnetic and gravitational phenomena on an equal footing with both being intimately tied to the curvature of space-time. In the development within, Maxwell's homogeneous equation was first shown to be a derivable consequence of equation (1). Next, Maxwell's inhomogeneous equation and the definition of charge density in terms of curved space-time were derived from equations (1) and (2) under the assumption that $a^{\lambda} R_{\lambda}{ }^{v}$ is time-like. The entirety of classical electromagnetism, including for example the Lorentz force law, was then shown to be a derivable consequence of fundamental equations (1) through (3). The theory of electromagnetism based on equations (1) through (3) that emerged and was shown to fully contain the equations of classical electromagnetism was then shown to go further with all electromagnetic phenomena being explained in terms of curved space-time. Next, the theory of gravitation emerging from the proposed theory was shown to be equivalent to Einstein's equation of General Relativity augmented by a term that can mimic the properties of dark matter and/or dark energy. Using specific solutions to the proposed theory, the unification brought to electromagnetic and gravitational phenomena as well as the consistency of those solutions with those of the classical M\&EFEs were emphasized. Also emphasized were unique features of the proposed theory that set it apart from the classical M\&EFEs. These distinguishing features include the emergence of antimatter and its behavior in electromagnetic and gravitational fields, the emergence of dark matter and dark energy mimicking terms in the context of General Relativity, an underlying relationship between electromagnetic and gravitational radiation, and the impossibility of negative mass solutions that would generate repulsive gravitational fields or antigravity. Although not yet based on specific solutions to the proposed theory, a method for quantizing the charge, mass, and angular momentum of particle-like solutions, as well as the possibility of superluminal transport when $a^{\lambda} R_{\lambda}{ }^{v}$ is space-like were conjectured.

The genesis of the work presented here was reported in a preliminary form in reference [2]. The same fundamental field equations proposed here were first reported there, although in a somewhat modified form. Also, the discussion of systems of first order partial differential equations and the existence of solutions to such systems was given in reference [2] but is included here to keep the mathematical description of the proposed theory self-contained. New to this manuscript is the discussion of the global 
symmetries of the fundamental field equations and based on those global symmetries the interpretation of the particle-like solution has been advanced, as has the discussion of boundary conditions. The derivation of Einstein's equation of General Relativity augmented by a term that can mimic the properties of dark matter and/or dark energy is also new to this manuscript, as is the discussion of the cosmological solution based on the FLRW metric. The present manuscript also corrects an error in the weak field analysis of reference [2], leading to the expanded discussion of electromagnetic radiation and its underlying gravitational radiation. The discussion of the impossibility of both negative mass solutions and antigravity is new. The speculation on superluminal transport being part of solutions if $a^{\lambda} R_{\lambda}{ }^{v}$ is space-like is also new. Finally, the appendix containing the analysis of the Cauchy initial value problem as it relates to the theory's fundamental field equations (1) through (3) is new and included to replace an incorrect discussion of the logical consistency of the fundamental field equations that was given in reference [2].

\section{ACKNOWLEDGEMENTS}

For his penetrating insights and suggestions offered during the early phases of this work ${ }^{[34]}$ I would like to express my appreciation and thanks to David Eimerl. For their many useful conversations and critical comments during the preparation of this manuscript I would like to thank my colleagues Charles Boley and Alexander Rubenchik. This work was performed under the auspices of the U.S. Department of Energy by Lawrence Livermore National Laboratory under Contract DE-AC52-07NA27344.

\section{APPENDIX - The Cauchy problem applied to the fundamental field equations}

One of the unusual features of fundamental field equations (1) through (3) is the lack of any explicit derivatives of the vector field $a^{\lambda}$, a situation which raises questions about the time dependent development of $a^{\lambda}$. To further elucidate this and other questions regarding solutions of the fundamental field equations, and to outline how they can be solved numerically, they are here analyzed in terms of a Cauchy initial value problem. For specificity, $a^{\lambda} R_{\lambda}{ }^{v}$ is assumed to be time-like in this section so that the concept of charge density $\rho_{c}$ is meaningful.

Given a set of initial conditions comprising the values of the fundamental fields in Table I at all spatial locations, a procedure is outlined that propagates those fields to any other time. To begin, assume 
$g_{\mu \nu}, F_{\mu \nu}, u^{\lambda}, \rho_{c}, \rho_{m}$ and $\frac{\partial g_{\mu \nu}}{\partial t}$ are known at all spatial coordinates at some initial coordinate time $t_{0}$. Note that the initial values for the field $a^{\lambda}$ are not required, rather they will be solved for using equation (1) as described below. Also note that in addition to $g_{\mu v}$ the initial values of $\frac{\partial g_{\mu \nu}}{\partial t}$ must be specified because the fundamental field equations are second order in the time derivatives of $g_{\mu \nu}$, a situation analogous to classical General Relativity. The goal of the Cauchy method as it applies here is to start with specified initial conditions for $g_{\mu \nu}, F_{\mu \nu}, u^{\lambda}, \rho_{c}, \rho_{m}$ and $\frac{\partial g_{\mu \nu}}{\partial t}$ at $t_{0}$, and then using the fundamental field equations (1) through (3) solve for $a^{\lambda}, R_{\lambda \kappa \mu v}, \frac{\partial F_{\mu \nu}}{\partial t}, \frac{\partial u^{\lambda}}{\partial t}, \frac{\partial \rho_{m}}{\partial t}, \frac{\partial \rho_{c}}{\partial t}$ and $\frac{\partial^{2} g_{\mu \nu}}{\partial t^{2}}$ at $t_{0}$. Armed with these values at $t_{0}$, it is straight forward to propagate the fields $g_{\mu \nu}, F_{\mu \nu}, u^{\lambda}, \rho_{c}, \rho_{m}$ and $\frac{\partial g_{\mu \nu}}{\partial t}$ from their initial conditions at $t_{0}$ to $t_{0}+d t$ and then solve for $a^{\lambda}, R_{\lambda \kappa \mu \nu}, \frac{\partial F_{\mu \nu}}{\partial t}, \frac{\partial u^{\lambda}}{\partial t}, \frac{\partial \rho_{m}}{\partial t}, \frac{\partial \rho_{c}}{\partial t}$ and $\frac{\partial^{2} g_{\mu \nu}}{\partial t^{2}}$ at $t_{0}+d t$ using the same procedure that was used to find them at $t_{0}$. Repeating this procedure, values for the fundamental fields of the theory can then be found at all times. One additional requirement on the field values specified by initial conditions is that they must be self-consistent with the fundamental field equations (1) through (3), i.e., the specified initial conditions must be consistent with a solution to the fundamental field equations (1) through (3).

In what follows, Greek indices $(\mu, v, \kappa, \ldots)$ take on the usual space-time coordinates 1-4 but Latin indices $(i, j, k, \ldots)$ are restricted to spatial coordinates, 1-3 only. Since the values of $g_{\mu v}$ and $\frac{\partial g_{\mu v}}{\partial t}$ are known at all spatial coordinates at time $t_{0}$, the values of $\frac{\partial g_{\mu \nu}}{\partial x^{i}}, \frac{\partial^{2} g_{\mu \nu}}{\partial x^{i} \partial x^{j}}$ and $\frac{\partial^{2} g_{\mu \nu}}{\partial x^{i} \partial t}$ can be calculated at all spatial coordinates at time $t_{0}$. This leaves the ten quantities $\frac{\partial^{2} g_{\mu \nu}}{\partial t^{2}}$ as the only second derivatives of $g_{\mu \nu}$ not known at $t_{0}$. To find the values of $\frac{\partial^{2} g_{\mu v}}{\partial t^{2}}$ at $t_{0}$ proceed as follows. First find the values of the six $\frac{\partial^{2} g_{i j}}{\partial t^{2}}$ at $t_{0}$ using a subset of equations from (1), the subset containing only those equations having spatial 
derivatives of $F_{\mu v}$ on the LHS and at most one time-index in each occurrence of the R-C tensor on the RHS. These equations will be used to solve for the values of $a^{\lambda}$ at time $t_{0}$. In all there are 12 such equations out of the 24 that comprise (1), as listed here:

$$
\begin{aligned}
& F_{12 ; 1}=a^{\lambda} R_{\lambda 112} \\
& F_{13 ; 1}=a^{\lambda} R_{\lambda 113} \\
& F_{23 ; 1}=a^{\lambda} R_{\lambda 123} \\
& F_{12 ; 2}=a^{\lambda} R_{\lambda 212} \\
& F_{13 ; 2}=a^{\lambda} R_{\lambda 213} \\
& F_{23 ; 2}=a^{\lambda} R_{\lambda 223} \\
& F_{12 ; 3}=a^{\lambda} R_{\lambda 312} \\
& F_{13 ; 3}=a^{\lambda} R_{\lambda 313} \\
& F_{23 ; 3}=a^{\lambda} R_{\lambda 323} \\
& F_{12 ; 4}=-F_{24 ; 1}-F_{41 ; 2}=a^{\lambda} R_{\lambda 412} \\
& F_{13 ; 4}=-F_{34 ; 1}-F_{41 ; 3}=a^{\lambda} R_{\lambda 413} \\
& F_{23 ; 4}=-F_{34 ; 2}-F_{42 ; 3}=a^{\lambda} R_{\lambda 423}
\end{aligned}
$$

The last three equations in (91) use (7), Maxwell's homogeneous equation to express the time derivative of a Maxwell tensor component on the LHS as the sum of the spatial derivatives of two Maxwell tensor components. The importance of having only spatial derivatives of the Maxwell tensor components on the LHS of (91) is that they are all known quantities at time $t_{0}$, i.e., since all the $F_{\mu \nu}$ are known at time $t_{0}$, all $\frac{\partial F_{\mu \nu}}{\partial x^{i}}$ and $F_{\mu v ; i}$ can be calculated at time $t_{0}$. Equally important is that the RHS of the 12 equations that comprise (91) contain at most a single time index in each occurrence of their R-C tensor and so are also known at time $t_{0}$. To see that this is so I examine the general form of the R-C tensor in a locally inertial coordinate system where all first derivatives of $g_{\mu \nu}$ vanish, i.e.,

$$
R_{\lambda \kappa \mu \nu}=\frac{1}{2}\left(\frac{\partial^{2} g_{\mu \lambda}}{\partial x^{v} \partial x^{\kappa}}-\frac{\partial^{2} g_{\mu \kappa}}{\partial x^{v} \partial x^{\lambda}}-\frac{\partial^{2} g_{v \lambda}}{\partial x^{\mu} \partial x^{\kappa}}+\frac{\partial^{2} g_{\kappa \nu}}{\partial x^{\mu} \partial x^{\lambda}}\right)
$$


Note, having at most a single time index on the RHS of (92) means that the R-C tensor is made up entirely of terms from $\frac{\partial^{2} g_{\mu \nu}}{\partial x^{i} \partial x^{j}}$ and $\frac{\partial^{2} g_{\mu \nu}}{\partial x^{i} \partial t}$, all of which are known at time $t_{0}$. Examining the set of equations (91) there are 12 equations for 4 unknowns, the unknowns being the components of $a^{\lambda}$. These 12 equations can be solved for $a^{\lambda}$ at time $t_{0}$ if the initial conditions were chosen self-consistently with the fundamental field equations (1) through (3), i.e., chosen such that a solution to the field equations is indeed possible.

Knowing the R-C tensor components with at most one time-index at $t_{0}$, I now proceed to determine the $\mathrm{R}-\mathrm{C}$ tensor components with two time indices. Going back to the 24 equations that comprise the set of equations (1), here I collect the subset of those equations in which the LHS is known at time $t_{0}$, i.e., contains only spatial derivatives of the Maxwell tensor, and the RHS has an R-C tensor component that contain two time indices:

$$
\begin{aligned}
& F_{14 ; 1}=a^{\lambda} R_{\lambda 114} \\
& F_{24 ; 1}=a^{\lambda} R_{\lambda 124} \\
& F_{34 ; 1}=a^{\lambda} R_{\lambda 134} \\
& F_{14 ; 2}=a^{\lambda} R_{\lambda 214} \\
& F_{24 ; 2}=a^{\lambda} R_{\lambda 224} \\
& F_{34 ; 2}=a^{\lambda} R_{\lambda 234} \\
& F_{14 ; 3}=a^{\lambda} R_{\lambda 314} \\
& F_{24 ; 3}=a^{\lambda} R_{\lambda 324} \\
& F_{34 ; 3}=a^{\lambda} R_{\lambda 334}
\end{aligned}
$$

Each of the equations in (93) contains only one unknown, the R-C component having two time indices. In total, there are six such independent $\mathrm{R}-\mathrm{C}$ tensor components:

$$
\begin{aligned}
& R_{1414} \\
& R_{1424} \\
& R_{1434} \\
& R_{2424} \\
& R_{2434} \\
& R_{3434}
\end{aligned}
$$


so the system of nine equations (93) can be algebraically solved for the these six unknown R-C components at time $t_{0}$. With this I now know the value of all components of the R-C tensor at time $t_{0}$. From the $t_{0}$ values of the R-C tensor components listed in (94), the values of the six unknown $\frac{\partial^{2} g_{i j}}{\partial t^{2}}$ at $t_{0}$ can be found.

There are three remaining equations from the set of equations (1) that have not yet been addressed:

$$
\begin{aligned}
& F_{14 ; 4}=a^{\lambda} R_{\lambda 414} \\
& F_{24 ; 4}=a^{\lambda} R_{\lambda 424} \\
& F_{34 ; 4}=a^{\lambda} R_{\lambda 434}
\end{aligned}
$$

These are the equations for which the temporal derivatives of the Maxwell tensor components are not yet known. Because all values of the R-C tensor and $a^{\lambda}$ are now known at $t_{0}$, these three remaining timedifferentiated components of the Maxwell tensor can now be solved for directly using (95), giving complete knowledge of $\frac{\partial F_{\mu v}}{\partial t}$ at time $t_{0}$.

If the values of the four $\frac{\partial^{2} g_{\mu 4}}{\partial t^{2}}$ could be calculated then all $\frac{\partial^{2} g_{\mu \nu}}{\partial t^{2}}$ would be known and all $\frac{\partial g_{\mu \nu}}{\partial t}$ could be propagated from $t_{0}$ to $t_{0}+d t$. Just as is the case with classical General Relativity, the four $\frac{\partial^{2} g_{\mu 4}}{\partial t^{2}}$ can be determined from the four coordinate conditions that are fixed by the choice of coordinate system. ${ }^{\text {[35] }}$ Recapping, at $t_{0}$ the following quantities are now known: $g_{\mu \nu}, F_{\mu \nu}, u^{\lambda}, \rho_{c}, \rho_{m}$ and $\frac{\partial g_{\mu \nu}}{\partial t}$ are defined by initial conditions, and $a^{\lambda}, \frac{\partial^{2} g_{\mu \nu}}{\partial x^{\kappa} \partial x^{\lambda}}, R_{\lambda \kappa \mu \nu}$, and $\frac{\partial F_{\mu \nu}}{\partial x^{\lambda}}$ are solved for using those initial conditions, the fundamental field equations, and the four coordinate conditions that are fixed by the choice of coordinate system. Still needed to propagate the initial conditions in time from $t_{0}$ to $t_{0}+d t$ are $\frac{\partial u^{\mu}}{\partial t}, \frac{\partial \rho_{m}}{\partial t}$ and $\frac{\partial \rho_{c}}{\partial t}$. Using the Lorentz force law (23), the following development, 


$$
\begin{gathered}
\rho_{m} \frac{D u^{\mu}}{D \tau}=\rho_{c} u^{\lambda} F_{\lambda}^{\mu} \\
\downarrow \\
\rho_{m} u_{; \nu}^{\mu} u^{v}=\rho_{c} u^{\lambda} F_{\lambda}^{\mu} \\
\downarrow \\
\rho_{m} u_{; 4}^{\mu} u^{4}=-\rho_{m} u_{; i}^{\mu} u^{i}+\rho_{c} u^{\lambda} F_{\lambda}^{\mu} \\
\downarrow \\
\rho_{m}\left(\frac{\partial u^{\mu}}{\partial t}+\Gamma_{4 \sigma}^{\mu} u^{\sigma}\right) u^{4}=-\rho_{m} u_{; i}^{\mu} u^{i}+\rho_{c} u^{\lambda} F_{\lambda}^{\mu}
\end{gathered}
$$

shows on the last line above that $\frac{\partial u^{\mu}}{\partial t}$ can be solved for at $t_{0}$ in terms of knowns at $t_{0}$. Using the conservation of mass (22) and knowing $\frac{\partial u^{\mu}}{\partial t}$ at $t_{0}$, the following development,

$$
\begin{gathered}
\left(\rho_{m} u^{v}\right)_{; v}=0 \\
\downarrow \\
\left(\rho_{m} u^{4}\right)_{; 4}=-\left(\rho_{m} u^{i}\right)_{; i} \\
\downarrow \\
\frac{\partial \rho_{m}}{\partial t} u^{4}=-\rho_{m} u_{; 4}^{4}-\left(\rho_{m} u^{i}\right)_{; i}
\end{gathered}
$$

shows on the last line above that $\frac{\partial \rho_{m}}{\partial t}$ can be solved for at $t_{0}$ in terms of knowns at $t_{0}$. Following an analogous development for $\rho_{c}$ using the charge conservation equation (18), $\frac{\partial \rho_{c}}{\partial t}$ can be solved for at $t_{0}$ in terms of knowns at $t_{0}$, or alternatively given using either equations (14) or (15). With these, the values of $a^{\lambda}, R_{\lambda \kappa \mu v}, \frac{\partial F_{\mu v}}{\partial t}, \frac{\partial u^{\lambda}}{\partial t}, \frac{\partial \rho_{m}}{\partial t}, \frac{\partial \rho_{c}}{\partial t}$ and $\frac{\partial^{2} g_{\mu v}}{\partial t^{2}}$ are all known at $t_{0}$ and can be used to propagate the initial conditions $g_{\mu \nu}, F_{\mu \nu}, u^{\lambda}, \rho_{c}, \rho_{m}$ and $\frac{\partial g_{\mu v}}{\partial t}$ at $t_{0}$ to time $t_{0}+d t$. Iterating the process, the values of the fundamental fields can be determined at all times. 


\section{REFERENCES AND END NOTES}

${ }^{[1]}$ For an historical review including brief technical descriptions of such theories in the early part of the $20^{\text {th }}$ century see: Hubert F. M. Goenner, “On the History of Unified Field Theories”, Living Rev. Relativity, 7, (2004), Online Article: cited Jan 10, 2012, http://www.livingreviews.org//rr-2004-2.

[2] Beach, R. (2014) A Classical Field Theory of Gravity and Electromagnetism. Journal of Modern Physics, 5, $928-939$. doi: 10.4236/jmp.2014.510096, and http://export.arxiv.org/abs/1102.2170v10.

${ }^{[3]}$ S. Weinberg, Gravitation and Cosmology, John Wiley \& Sons, New York, NY 1972. The definition of the RiemannChristoffel curvature tensor is $R_{\mu \nu \kappa}^{\lambda} \equiv \partial_{\kappa} \Gamma_{\mu \nu}^{\lambda}-\partial_{\nu} \Gamma_{\mu \kappa}^{\lambda}+\Gamma_{\mu \nu}^{\eta} \Gamma_{\kappa \eta}^{\lambda}-\Gamma_{\mu \kappa}^{\eta} \Gamma_{\nu \eta}^{\lambda}$, and the definition of the Ricci tensor is $R_{\mu \kappa} \equiv R_{\mu \lambda \kappa}^{\lambda}$.

${ }^{[4]}$ See for example: D. E. Soper, Classical Field Theory, Ch 8 The Electromagnetic Field, Dover Edition, 2008, and S. Weinberg, Gravitation and Cosmology, Ch 12 The Action Principle, John Wiley \& Sons, New York, NY 1972.

${ }^{[5]}$ A. A. Chernitskii, "On Unification of Gravitation and Electromagnetism in the Framework of a General-Relativistic Approach," ISSN 0202-2893, Gravitation and Cosmology, Vol. 15, No. 2, (2009) pp. 151-153.

${ }^{[6]}$ Chang, Yi-Fang. "Unification of gravitational and electromagnetic fields in Riemannian geometry." arXiv preprint arXiv:0901.0201 (2009).

[7] Jun Wang, Ling. "Unification of gravitational and electromagnetic fields." Physics Essays 31, no. 1 (2018): $81-88$.

${ }^{[8]}$ Fasihi-Ramandi, Gh. "Semi-symmetric connection formalism for unification of gravity and electromagnetism." Journal of Geometry and Physics 144 (2019): 245-250.

${ }^{[9]}$ Li, L.X., 2016. A new unified theory of electromagnetic and gravitational interactions. Frontiers of Physics, 11(6), p.110402.

[10] This section follows the discussion given in reference [2] and is included here to keep the mathematical description of the proposed theory self-contained.

${ }^{[11]}$ L.P. Eisenhart, An Introduction to Differential Geometry, Chapter 23, "Systems of Partial Differential Equations of the First Order, Mixed Systems," Princeton University Press 1947.

[12] The interpretation of the solution here is different than in the derivation in reference [2]. Specifically, in reference [2] the charge density was restricted to be positive, a restriction that is lifted here.

${ }^{[13]}$ C. Misner, K. Thorne, and J. Wheeler, Gravitation, p. 840, W.H. Freeman and Company, San Francisco, CA 1970

${ }^{[14]}$ Calculations within were performed in Mathematica: Wolfram Research, Inc., Mathematica, Version 12.1, Champaign, IL (2020).

[15] This calculation was presented in reference [2] but contained an error that is corrected here. In [2], the electric and magnetic fields where not restricted to the same weak field approximation as the $h$ 's.

${ }^{[16]}$ S. Weinberg, Gravitation and Cosmology, John Wiley \& Sons, New York, NY 1972, section 10.2.

${ }^{[17]}$ L.D. Landau and E.M. Lifshitz, The Classical Theory of Fields, Fourth Edition, p. 235, Pergamon Press, New York, NY 1975, section 107.

${ }^{[18]}$ S. Weinberg, Gravitation and Cosmology, John Wiley \& Sons, New York, NY 1972, Chapter 13, "Symmetric Spaces".

[19] Tkachev, Igor. "Cosmology and Dark Matter." arXiv preprint arXiv:1802.02414 (2018).

[20] Huterer, Dragan, and Daniel L. Shafer. "Dark energy two decades after: Observables, probes, consistency tests." Reports on Progress in Physics 81, no. 1 (2017): 016901.

${ }^{[21]}$ Starkman, Glenn D. "Modifying gravity: you cannot always get what you want." Phil. Trans. R. Soc. A 369, no. 1957 (2011): 5018-5041.

[22] Schmidt, Fabian. "Reining in Alternative Gravity." Physics 10 (2017): 134.

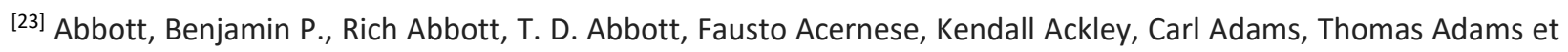
al. "GW170817: observation of gravitational waves from a binary neutron star in spiral." Physical Review Letters 119, no. 16 (2017): 161101. 
${ }^{[24]}$ Goldstein, A., Veres, P., Burns, E., Briggs, M.S., Hamburg, R., Kocevski, D., Wilson-Hodge, C.A., Preece, R.D., Poolakkil, S., Roberts, O.J., and Hui, C.M., 2017. An ordinary short gamma-ray burst with extraordinary implications: Fermi-GBM detection of GRB 170817A. The Astrophysical Journal Letters, 848(2), p.L14.

${ }^{[25]}$ R.P. Feynman, "The Theory of Positrons," Phys Rev, 1949, 76(6): p. 749-759.

${ }^{[26]}$ Bondi, Hermann. "Negative mass in general relativity." Reviews of Modern Physics 29, no. 3 (1957): 423.

${ }^{[27]}$ Bonnor, W. B. "Negative mass in general relativity." General relativity and gravitation 21, no. 11 (1989): 11431157.

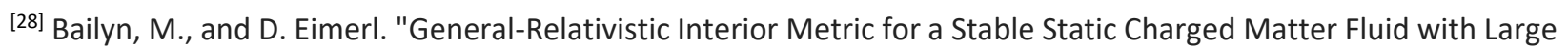
e m." Physical Review D 5, no. 8 (1972): 1897.

[29] Bonnor, W.B. and Cooperstock, F.I., 1989. Does the electron contain negative mass? Physics Letters A, 139(9), pp.442-444.

${ }^{[30]}$ Herrera, L., and V. Verela. "Negative energy density and classical electron models." Physics Letters A 189, no. 1-2 (1994): 11-14.

${ }^{[31]}$ L.D. Landau and E.M. Lifshitz, The Classical Theory of Fields, Fourth Edition, p. 235, Pergamon Press, New York, NY 1975.

${ }^{[32]}$ Blinder, S. M. "General relativistic models for the electron." Reports on Mathematical Physics 47.2 (2001): $279-$ 285.

[33] Popescu, Sandu. "Nonlocality beyond quantum mechanics." Nature Physics 10, no. 4 (2014): 264-270.

[34] Beach, Raymond J. "A Representation of the Electromagnetic Field in the Presence of Curvature." Physics Essays 12 (1999): 457.

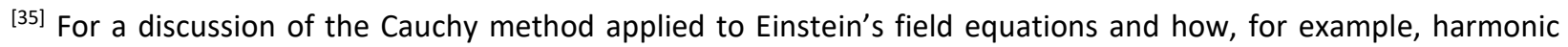
coordinate conditions determine $\frac{\partial^{2} g_{\mu 4}}{\partial t^{2}}$ see, "The Cauchy Problem," section 5.5 of S. Weinberg, Gravitation and Cosmology, John Wiley \& Sons, New York, NY 1972. 